\title{
GROUP FOLIATION OF DIFFERENTIAL EQUATIONS USING MOVING FRAMES
}

\author{
ROBERT THOMPSON ${ }^{1}$ and FRANCIS VALIQUETTE ${ }^{2}$ \\ ${ }^{1}$ Department of Mathematics, Carleton College, Northfield, MN 55057, USA; \\ email: rthompson@carleton.edu \\ ${ }^{2}$ Department of Mathematics, SUNY at New Paltz, New Paltz, NY 12561, USA
}

Received 1 August 2014; accepted 11 September 2015

\begin{abstract}
We incorporate the new theory of equivariant moving frames for Lie pseudogroups into Vessiot's method of group foliation of differential equations. The automorphic system is replaced by a set of reconstruction equations on the pseudogroup jets. The result is a completely algorithmic and symbolic procedure for finding both invariant and noninvariant solutions of differential equations admitting a symmetry group.
\end{abstract}

2010 Mathematics Subject Classification: 35B06, 53A55 (primary); 35C05, 58H05 (secondary)

\section{Introduction}

The method of group foliation (also called group splitting, or group stratification) is a procedure for obtaining solutions of differential equations invariant under a symmetry group. The idea was proposed by Lie [27], and subsequently developed by Vessiot [56]. Later work of Johnson, Ovsiannikov, and others $[9,19,47]$ showed renewed interest. More recently, group foliation has been used to study equations of mathematical physics $[30,34]$, and reformulated using the language of exterior differential systems [3], demonstrating potential for further development and application.

Consider a differential equation $\Delta=0$ with symmetry group $\mathcal{G}$, possibly infinite dimensional. The method of group foliation uses a foliation of the solution space of $\Delta=0$ by the orbits of the group action to decompose $\Delta=0$ into two alternative systems of differential equations, called the resolving and automorphic

(c) The Author(s) 2015. This is an Open Access article, distributed under the terms of the Creative Commons Attribution licence (http://creativecommons.org/licenses/by/4.0/), which permits unrestricted re-use, distribution, and reproduction in any medium, provided the original work is properly cited. 


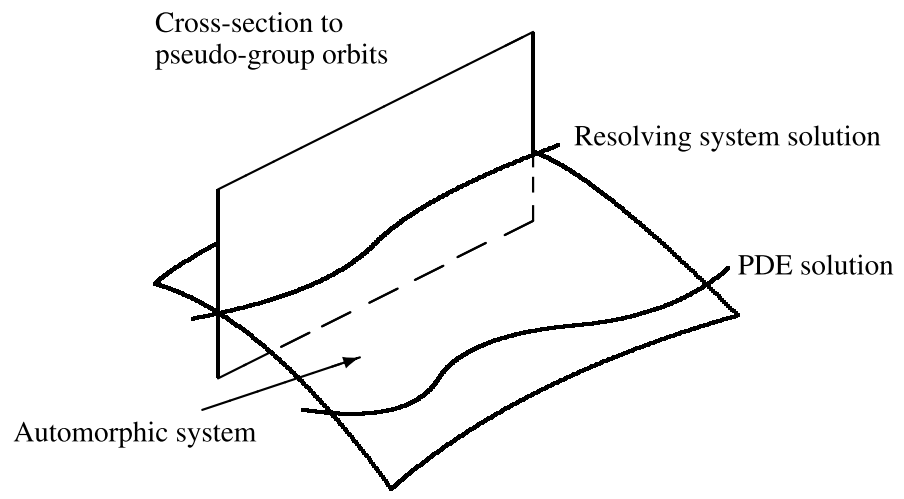

Figure 1. The geometry of group foliation.

systems. An automorphic system, characterized by the property that all solutions are situated on a single orbit of $\mathcal{G}$, describes the leaves of the foliation. The resolving system links the original differential equation to a specific automorphic system in the sense that each resolving system solution specifies a leaf of the foliation. See Figure 1 for the geometry of this construction. Application of group foliation may roughly be understood as a process of removing symmetries; quoting Ovsiannikov [47]:

The practical significance of group splitting consists in the fact that solutions of the automorphic system are very simply found at the expense of its automorphic property (by operation with a group transformation on any of its solutions), and the resolving system turns out to be simple when compared with the initial equation $\Delta=0$. The latter occurs because the resolving system has fewer solutions than $\Delta=0$ does because of removal of those excesses which were introduced by the existence of the admitted group $\mathcal{G}$.

Our main tool will be the theory of equivariant moving frames $[13,43$, 44]. The determination of the resolving system relies on the classification of differential invariants and their syzygies, which may be performed algorithmically using the universal recurrence relation (32). The resolving system may be interpreted as a projection of the original differential equation into a space of invariants, accomplished through the application of a right moving frame. The automorphic system then provides a method for reconstructing solutions to the original differential equation from resolving system solutions. Geometrically, this reconstruction process is the reversal of the right moving frame projection, accomplished by application of a left moving frame. 
Our approach was inspired by Mansfield's use of equivariant moving frames to solve ordinary differential equations $[28, \mathrm{Ch} .7]$. This approach works for Lie group actions, and it relies on the choice of a faithful matrix representation for the group. In this paper, we adapt these constructions to infinite-dimensional Lie pseudogroup actions. Central to this adaptation is the introduction of the pseudogroup jet differential expressions which, after pull-back by a moving frame, generalize Cartan's structure equations of a moving frame for Lie group actions [14], and play the role of Mansfield's 'curvature matrix' equation in the reconstruction process. The reconstruction step is also related to the reconstruction procedure appearing in symmetry reduction of exterior differential systems $[2,3,48]$.

In its most general formulation, the group foliation method applies to infinitedimensional Lie pseudogroup actions, so we begin by reviewing in Section 2 the basics of Lie pseudogroups. The theory of equivariant moving frames is introduced in Section 3. We begin our discussion of group foliation in Section 4.1. In Section 4.2, we incorporate the moving frame apparatus and obtain a new perspective-in particular, a natural geometric approach to the reconstruction step-based on moving frames. The Lie pseudogroup action

$$
X=f(x), \quad Y=y, \quad U=\frac{u}{f^{\prime}(x)}
$$

considered in $[41,43]$, is used as a running example for our constructions. This pseudogroup is also used in [48] to illustrate the method of symmetry reduction of exterior differential systems admitting an infinite-dimensional symmetry group; we reproduce these results in Examples 28 and 47. In Section 5, the group foliation method is applied to several equations of interest, including a nonlinear wave equation studied by Calogero [5], the equation of a transonic gas flow, and a nonlinear second-order ordinary differential equation. Finally, when a symmetry pseudogroup $\mathcal{G}$ admits a chain of normal subpseudogroups, we explain in Section 6 how the reconstruction procedure splits into a sequence of smaller reconstruction problems.

\section{Lie pseudogroups}

Since we work with infinite-dimensional Lie pseudogroup actions, we restrict our considerations to the analytic category. Given an analytic $m$-dimensional manifold $M$, let $\mathcal{D}=\mathcal{D}(M)$ denote the pseudogroup of all local diffeomorphisms $\varphi: M \rightarrow M$. For each $n \geqslant 0$, we denote by $\mathcal{D}^{(n)}$ the subbundle formed by their $n$ th-order jets $\mathrm{j}_{n} \varphi$. Introducing the local coordinates $Z=\varphi(z)$ on $\mathcal{D}^{(0)}=M \times M$, we denote by $z=\widetilde{\boldsymbol{\sigma}}\left(\mathrm{j}_{n} \varphi\right)$ and $Z=\widetilde{\boldsymbol{\tau}}\left(\mathrm{j}_{n} \varphi\right)$ the source and target coordinates of $\varphi$. 
The induced coordinates on $\mathcal{D}^{(n)}$ are $\mathrm{j}_{n} \varphi=\left(z, Z^{(n)}\right)$, where $Z^{(n)}$ indicates the derivatives

$$
Z_{A}^{b}=\frac{\partial^{k} Z^{b}}{\partial z^{a_{1}} \cdots \partial z^{a_{k}}}, \quad b=1, \ldots, m, \quad A=\left(a_{1}, \ldots, a_{k}\right),
$$

of order $0 \leqslant k \leqslant n$. A local diffeomorphism $\psi \in \mathcal{D}$ acts on $\mathcal{D}^{(n)}$ by either left or right multiplication:

$$
\mathrm{L}_{\psi}\left(\left.\mathrm{j}_{n} \varphi\right|_{z}\right)=\left.\mathrm{j}_{n}(\psi \circ \varphi)\right|_{z} \quad \text { or } \quad \mathrm{R}_{\psi}\left(\left.\mathrm{j}_{n} \varphi\right|_{z}\right)=\left.\mathrm{j}_{n}\left(\varphi \circ \psi^{-1}\right)\right|_{\psi(z)} .
$$

The definition of a pseudogroup $\mathcal{G} \subset \mathcal{D}$ is a natural extension of the concept of a local Lie group action. We refer to [17] for a precise definition.

DEFINITION 1. A pseudogroup $\mathcal{G} \subset \mathcal{D}$ is called a Lie pseudogroup of order $n^{\star} \geqslant 1$ if, for all finite $n \geqslant n^{\star}$, the following hold.

- The pseudogroup jets $\tilde{\sigma}: \mathcal{G}^{(n)} \rightarrow M$ form an embedded subbundle of $\tilde{\boldsymbol{\sigma}}: \mathcal{D}^{(n)} \rightarrow M$.

- The projection $\pi_{n}^{n+1}: \mathcal{G}^{(n+1)} \rightarrow \mathcal{G}^{(n)}$ is a fibration.

- Every local diffeomorphism $\varphi \in \mathcal{D}$ satisfying $\mathrm{j}_{n^{\star}} \varphi \subset \mathcal{G}^{\left(n^{\star}\right)}$ belongs to $\mathcal{G}$.

The above regularity conditions imply that, in some coordinate chart, the subbundle $\mathcal{G}^{\left(n^{\star}\right)}$ is described by a system of $n^{\star}$ th-order differential equations

$$
F^{\left(n^{\star}\right)}\left(z, Z^{\left(n^{\star}\right)}\right)=0
$$

called the determining system of $\mathcal{G}$. For $n \geqslant n^{\star}, \mathcal{G}^{(n)}$ is described by the prolongation of (2).

EXAMPLE 2. As our running example, we consider the Lie pseudogroup action

$$
X=f(x), \quad Y=y, \quad U=\frac{u}{f^{\prime}(x)}, \quad f \in \mathcal{D}(\mathbb{R}),
$$

on $M=\mathbb{R}^{3} \backslash\{u=0\}$, defined by the system of differential equations

$$
X_{y}=X_{u}=0, \quad Y=y, \quad U=\frac{u}{X_{x}} .
$$

As is generally the case, it is preferable to work with the Lie algebra of infinitesimal generators of a Lie pseudogroup. Let $\mathcal{X}(M)$ denote the space of 
locally defined vector fields on $M$. In local coordinates, we use the notation

$$
\mathbf{v}=\sum_{a=1}^{m} \zeta^{a}(z) \frac{\partial}{\partial z^{a}}
$$

to denote a vector field. For $0 \leqslant n \leqslant \infty$, let $\mathrm{J}^{n} T M$ denote the $n$ th-order jet bundle of the tangent bundle with local coordinates $\mathrm{j}_{n} \mathbf{v}=\left(z, \zeta^{(n)}\right)$. Once more, $\zeta^{(n)}$ denotes the collection of derivatives $\zeta_{A}^{a}, a=1, \ldots, m, 0 \leqslant \# A \leqslant n$.

Given a Lie pseudogroup $\mathcal{G}$, let $\mathfrak{g} \subset \mathcal{X}(M)$ denote its Lie algebra consisting of local infinitesimal generators whose flows belong to the pseudogroup. A vector field (5) is in $\mathfrak{g}$ if its $n^{\star}$-jet is a solution of the linear system of partial differential equations

$$
L^{\left(n^{\star}\right)}\left(z, \zeta^{\left(n^{\star}\right)}\right)=0
$$

called the infinitesimal determining system of $\mathfrak{g}$, obtained by linearizing the determining system (2) at the identity jet. When $\mathcal{G}$ is the symmetry group of a differential equation, the infinitesimal determining system (6) is obtained by implementing Lie's algorithm for determining the infinitesimal symmetry generators [36].

EXAMPLE 3. The infinitesimal generators of the pseudogroup action (3) are

$$
\mathbf{v}=\xi \frac{\partial}{\partial x}+\eta \frac{\partial}{\partial y}+\phi \frac{\partial}{\partial u}=a(x) \frac{\partial}{\partial x}-u a_{x}(x) \frac{\partial}{\partial u},
$$

where $a(x)$ is an arbitrary analytic function. The coefficients of the vector field (7) are solutions to the infinitesimal determining system

$$
\xi_{y}=\xi_{u}=0, \quad \eta=0, \quad \phi=-u \xi_{x},
$$

obtained by linearizing the determining equations (4) at the identity jet $\mathbb{1}^{(1)}$. Relations among higher-order vector field jets are obtained by considering the prolongation of (8).

Dual to the Lie algebra $\mathfrak{g}$ are the $\mathcal{G}$-invariant Maurer-Cartan forms. Since these play an important role in what follows, we now recall the details of their construction [41]. Beginning with the diffeomorphism pseudogroup $\mathcal{D}$, we split the differential $d=d_{M}+d_{\mathcal{G}}$ into its horizontal and group (or vertical/contact) components, as is done in the standard variational bicomplex construction [1], and observe that this splitting is invariant under the pseudogroup multiplication (1). Since the target coordinates $Z^{a}$ are right invariant, the horizontal one-forms

$$
\sigma^{z^{a}}=d_{M} Z^{a}=\sum_{b=1}^{m} Z_{b}^{a} d z^{b}, \quad a=1, \ldots, m,
$$


are also right invariant. Let $\mathbb{D}_{Z^{1}}, \ldots, \mathbb{D}_{Z^{m}}$ be the dual right-invariant total derivative operators defined by

$$
d_{M} F=\sum_{a=1}^{m}\left(\mathbb{D}_{Z^{a}} F\right) \sigma^{z^{a}}, \quad \text { for } F: \mathcal{D}^{(\infty)} \rightarrow \mathbb{R}
$$

Explicitly,

$$
\mathbb{D}_{Z^{a}}=\sum_{b=1}^{m} w_{a}^{b} \mathbb{D}_{z^{b}}, \quad \text { where }\left(w_{a}^{b}\right)=\left(Z_{a}^{b}\right)^{-1}
$$

and

$$
\mathbb{D}_{z^{b}}=\frac{\partial}{\partial z^{b}}+\sum_{\# A \geqslant 0} Z_{A, b} \frac{\partial}{\partial Z_{A}}, \quad b=1, \ldots, m,
$$

are the total derivative operators on $\mathcal{D}^{(\infty)}$. Then, the right-invariant Maurer-Cartan forms are obtained by successively Lie differentiating the zero-order invariant contact forms

$$
\mu^{a}=d_{\mathcal{G}} Z^{a}=d Z^{a}-\sum_{b=1}^{m} Z_{b}^{a} d z^{b}
$$

with respect to (9):

$$
\mu_{A}^{a}=\mathbb{D}_{Z}^{A} \mu^{a}
$$

We denote by $\mu^{(n)}$ the set of right-invariant Maurer-Cartan forms of order $\leqslant n$.

For the implementation of the moving frame method, the coordinate expressions of the Maurer-Cartan forms are not required. It is enough to know that these invariant group forms exist since, in practice, most computations involving the Maurer-Cartan forms can be done symbolically.

Under the inclusion map $i: \mathcal{G}^{(\infty)} \hookrightarrow \mathcal{D}^{(\infty)}$, the pulled-back Maurer-Cartan forms $\mu_{A}^{a}=i^{*}\left(\mu_{A}^{a}\right)$ are no longer linearly independent. In the following, to simplify the notation, we systematically avoid writing pull-backs.

PROPOSITION 4. Let $\mathcal{G}$ be a Lie pseudogroup of order $n^{\star}$. Then, for all $n \geqslant n^{\star}$, the restricted Maurer-Cartan forms $\left.\mu^{(n)}\right|_{\mathcal{G}}$ satisfy the $n$ th-order lifted linear relations

$$
L^{(n)}\left(Z, \mu^{(n)}\right)=0,
$$

obtained from the infinitesimal determining system (6) and its prolongation by making the substitutions $z^{a} \rightarrow Z^{a}$ and $\zeta_{A}^{a} \rightarrow \mu_{A}^{a}$.

EXAMPLE 5. Continuing Example 3, the right-invariant Maurer-Cartan forms of the Lie pseudogroup (3) satisfy the linear relations

$$
\mu_{Y}^{x}=\mu_{U}^{x}=0, \quad \mu^{y}=0, \quad \mu^{u}=-U \mu_{X}^{x},
$$


obtained from the infinitesimal determining equations (8) by making the substitutions

$$
\xi_{A} \rightarrow \mu_{A}^{x}, \quad \eta_{A} \rightarrow \mu_{A}^{y}, \quad \phi_{A} \rightarrow \mu_{A}^{u} \quad \text { and } \quad x \rightarrow X, \quad y \rightarrow Y, \quad u \rightarrow U .
$$

Linear relations among the higher-order Maurer-Cartan forms are obtained by Lie differentiating (12) with respect to $\mathbb{D}_{X}, \mathbb{D}_{Y}, \mathbb{D}_{U}$. It follows that a basis of right-invariant Maurer-Cartan forms is given by $\mu_{k}=\mu_{X^{k}}^{x}, k \geqslant 0$.

By pseudogroup inversion, the preceding discussion also holds for the left multiplication (1). Denote the inverse of $Z=\varphi(z)$ by $z=\varphi^{-1}(Z)$. Then the above formulas may be adapted to the left action by interchanging the variables $z^{a}$ and $Z^{a}$. In particular, the left-invariant Maurer-Cartan forms are obtained by successively Lie differentiating

$$
\bar{\mu}^{a}=d z^{a}-\sum_{b=1}^{m} z_{Z^{b}}^{a} d Z^{b}
$$

with respect to

$$
\mathbb{D}_{z^{a}}=\sum_{b=1}^{m} W_{a}^{b} \mathbb{D}_{Z^{b}}, \quad \text { where }\left(W_{a}^{b}\right)=\left(z_{Z^{a}}^{b}\right)^{-1},
$$

so that

$$
\bar{\mu}_{A}^{a}=\mathbb{D}_{z}^{A} \bar{\mu}^{a} .
$$

For the implementation of the group foliation method it will be useful to know the relation between left-invariant and right-invariant Maurer-Cartan forms. For the order-zero Maurer-Cartan forms we find that

$$
\bar{\mu}^{a}=d z^{a}-\sum_{b=1}^{m} z_{Z^{b}}^{a} d Z^{b}=-\sum_{b=1}^{m} z_{Z^{b}}^{a}\left(d Z^{b}-\sum_{c=1}^{m} Z_{z^{c}}^{b} d z^{c}\right)=-\sum_{b=1}^{m} z_{Z^{b}}^{a} \mu^{b} .
$$

The linear relations among the higher-order Maurer-Cartan forms are obtained by Lie differentiating (14) with respect to (13):

$$
\bar{\mu}_{A}^{a}=-\sum_{b=1}^{m} \sum_{B \leqslant A}\left(\begin{array}{l}
A \\
B
\end{array}\right) \mathbb{D}_{z}^{B}\left(z_{Z^{b}}^{a}\right) \cdot \mathbb{D}_{z}^{A-B}\left(\mu^{b}\right) .
$$

For example, for the first-order Maurer-Cartan forms, we have the relations

$$
\bar{\mu}_{b}^{a}=\mathbb{D}_{z^{b}}\left(\bar{\mu}^{a}\right)=-\sum_{b, c=1}^{m} W_{b}^{c}\left(z_{Z^{b} Z^{c}}^{a} \mu^{b}+z_{Z^{b}}^{a} \mu_{c}^{b}\right) .
$$


For $\mathcal{G} \subset \mathcal{D}$, the relations between the left-invariant and right-invariant MaurerCartan forms are obtain by restricting (14), (15) to the determining system (2) and the lifted determining equations (11).

EXAMPLE 6. For our running example, formula (14) reduces to

$$
\begin{aligned}
\bar{\mu}=\bar{\mu}^{X} & =-\left[x_{X} \mu^{x}+x_{Y} \mu^{y}+x_{U} \mu^{u}\right]=-x_{X} \mu, \\
\bar{\mu}^{Y} & =-\left[y_{X} \mu^{x}+y_{Y} \mu^{y}+y_{U} \mu^{u}\right]=-\mu^{y}=0, \\
\bar{\mu}^{U} & =-\left[u_{X} \mu^{x}+u_{Y} \mu^{y}+u_{U} \mu^{u}\right]=\frac{u x_{X X}}{x_{X}} \mu^{x}-\frac{1}{x_{X}} \mu^{u}=\frac{u x_{X X}}{x_{X}} \mu-u \mu_{X},
\end{aligned}
$$

where we used (12) and the determining equations

$$
x_{Y}=x_{U}=0, \quad y=Y, \quad u=\frac{U}{x_{X}} .
$$

Lie differentiating (16) with respect to

$$
\mathbb{D}_{x}=\frac{1}{x_{X}} \mathbb{D}_{X}+\frac{u x_{X X}}{x_{X}} \mathbb{D}_{U}, \quad \mathbb{D}_{y}=\mathbb{D}_{Y}, \quad \mathbb{D}_{u}=x_{X} \mathbb{D}_{U}
$$

yields the relations among the higher-order Maurer-Cartan forms. For example,

$$
\begin{aligned}
\bar{\mu}_{x} & =\mathbb{D}_{x}(\bar{\mu})=-\frac{x_{X X}}{x_{X}} \mu-\mu_{X}, \\
\bar{\mu}_{x x} & =\mathbb{D}_{x}\left(\bar{\mu}_{x}\right)=\left(\frac{x_{X X}^{2}}{x_{X}^{3}}-\frac{x_{X X X}}{x_{X}^{2}}\right) \mu-\frac{x_{X X}}{x_{X}^{2}} \mu_{X}-\frac{1}{x_{X}} \mu_{X X} .
\end{aligned}
$$

\section{Moving frames}

We are interested in the action of a Lie pseudogroup $\mathcal{G}$ on $p$-dimensional submanifolds $S \subset M$, with $1 \leqslant p<m=\operatorname{dim} M$. To this end, let $\mathrm{J}^{n}=\mathrm{J}^{n}(M, p)$ denote the $n$ th-order extended jet bundle of equivalence classes of $p$-dimensional submanifolds under the equivalence relation of $n$ th-order contact [35]. Locally, we identify $M \simeq X \times U$ with the Cartesian product of the submanifolds $X$ and $U$ with local coordinates $z=(x, u)$. The coordinates $x=\left(x^{1}, \ldots, x^{p}\right)$ and $u=\left(u^{1}, \ldots, u^{q}\right)$ are considered as independent and dependent variables, respectively. This induces the local coordinates $z^{(n)}=\left(x, u^{(n)}\right)$ on $\mathrm{J}^{n}$, where $u^{(n)}$ denotes the collection of derivatives $u_{J}^{\alpha}$, with $\alpha=1, \ldots, q$ and $0 \leqslant \# J \leqslant n$.

We introduce the $n$ th-order lifted bundle

$$
\mathcal{E}^{(n)}=\mathbf{J}^{n} \times{ }_{M} \mathcal{G}^{(n)} \rightarrow \mathbf{J}^{n},
$$


whose local coordinates are given by $\left(z^{(n)}, g^{(n)}\right)$, where $z^{(n)}$ are the $n$ th-order submanifold jet coordinates and $g^{(n)}$ are the fiber coordinates along $\left.\mathcal{G}^{(n)}\right|_{z}$. On the infinite-order lifted bundle $\mathcal{E}^{(\infty)}$, define the total derivative operators

$$
D_{x^{i}}=\mathbb{D}_{x^{i}}+\sum_{\alpha=1}^{q}\left[u_{i}^{\alpha} \mathbb{D}_{u^{\alpha}}+\sum_{J} u_{J, i}^{\alpha} \frac{\partial}{\partial u_{J}^{\alpha}}\right], \quad i=1, \ldots, p,
$$

where the expressions for the differential operators $\mathbb{D}_{x^{i}}, \mathbb{D}_{u^{\alpha}}$ are given in (10).

A Lie pseudogroup $\mathcal{G}$ acts on $\mathrm{J}^{n}$ by the usual prolonged action

$$
\left(X, U^{(n)}\right)=g^{(n)} \cdot\left(x, u^{(n)}\right)=g^{(n)} \cdot \mathrm{j}_{n} S=\mathrm{j}_{n}(g \cdot S) .
$$

The coordinate expressions of the prolonged action are obtained by applying the lifted total derivative operators

$$
D_{X^{i}}=\sum_{j=1}^{p} B_{i}^{j} D_{x^{j}}, \quad \text { where }\left(B_{i}^{j}\right)=\left(D_{x^{i}} X^{j}\right)^{-1},
$$

to the dependent target coordinates $U^{\alpha}$ :

$$
U_{X^{J}}^{\alpha}=D_{X}^{J} U^{\alpha}, \quad \alpha=1, \ldots, q, \# J \geqslant 0 .
$$

The lifted bundle $\mathcal{E}^{(n)}$ has groupoid structure with source map

$$
\boldsymbol{\sigma}^{(n)}\left(z^{(n)}, g^{(n)}\right)=z^{(n)}
$$

given by the projection onto the first factor, and target map

$$
\boldsymbol{\tau}^{(n)}\left(z^{(n)}, g^{(n)}\right)=g^{(n)} \cdot z^{(n)}
$$

given by the prolonged action (17). On $\mathcal{E}^{(\infty)}$ we use $\sigma$ and $\boldsymbol{\tau}$ to denote the source and target maps.

EXAMPLE 7. The Lie pseudogroup (3) is now assumed to act on surfaces in $\mathbb{R}^{3}$ locally given as graphs $\{x, y, u(x, y)\}$. In this setting, the prolonged action is obtained by applying the lifted total derivative operators

$$
D_{X}=\frac{1}{f_{x}} D_{x}, \quad D_{Y}=D_{y},
$$

to $U$. For example, the second-order prolonged action is

$$
\begin{gathered}
U_{Y}=\frac{u_{y}}{f_{x}}, \quad U_{X}=\frac{u_{x} f_{x}-u f_{x x}}{f_{x}^{3}}, \quad U_{Y Y}=\frac{u_{y y}}{f_{x}} \\
U_{X Y}=\frac{u_{x y}-U_{Y} f_{x x}}{f_{x}^{2}}, \quad U_{X X}=\frac{u_{x x} f_{x}-u f_{x x x}}{f_{x}^{4}}-3 \frac{U_{X} f_{x x}}{f_{x}^{2}} .
\end{gathered}
$$


From this point forward, we assume that the Lie pseudogroup acts regularly on $\mathrm{J}^{n}$ for all $n$. This means that the orbits of the pseudogroup action form a regular foliation and that its leaves intersect small open sets in pathwise connected subsets.

\section{DEFINITION 8. Let}

$$
\mathcal{G}_{z^{(n)}}^{(n)}=\left\{\left.g^{(n)} \in \mathcal{G}^{(n)}\right|_{z}: g^{(n)} \cdot z^{(n)}=z^{(n)}\right\}
$$

be the isotropy subgroup of $z^{(n)}$. The pseudogroup $\mathcal{G}$ acts freely at $z^{(n)}$ if $\mathcal{G}_{z^{(n)}}^{(n)}=\left\{\mathbb{1}_{z}^{(n)}\right\}$. The pseudogroup $\mathcal{G}$ is said to act freely at order $n$ if it acts freely on an open subset $\mathcal{V}^{n} \subset \mathrm{J}^{n}$, called the set of regular $n$-jets.

DEFINITION 9. Let $\mathcal{G}$ be a Lie pseudogroup acting regularly and freely on $\mathcal{V}^{n} \subset \mathbf{J}^{n}$, and let $\mathcal{K}^{n} \subset \mathcal{V}^{n}$ be a local cross-section to the pseudogroup orbits. Given $z^{(n)} \in \mathcal{V}^{n}$, the $n$ th-order right moving frame

$$
\varrho^{(n)}\left(z^{(n)}\right)=\left(z^{(n)}, \rho^{(n)}\left(z^{(n)}\right)\right)
$$

is the section of the lifted bundle $\mathcal{E}^{(n)}$ where the fiber component $\rho^{(n)}\left(z^{(n)}\right)$ is the unique $n$ th-order pseudogroup jet in $\left.\mathcal{G}^{(n)}\right|_{z}$ such that

$$
\boldsymbol{\tau}^{(n)}\left[\varrho^{(n)}\left(z^{(n)}\right)\right]=\rho^{(n)}\left(z^{(n)}\right) \cdot z^{(n)} \in \mathcal{K}^{n} .
$$

Assuming, to simplify the discussion, that $\mathcal{K}^{n}$ is the coordinate cross-section

$$
x^{i_{1}}=c_{1}, \ldots, x^{i_{l}}=c_{l}, \quad u_{J_{l+1}}^{\alpha_{l+1}}=c_{l+1}, \ldots, u_{J_{d_{n}}}^{\alpha_{d_{n}}}=c_{d_{n}},
$$

where $d_{n}=\left.\operatorname{dim} \mathcal{G}^{(n)}\right|_{z}$ is the fiber dimension of the subbundle $\mathcal{G}^{(n)}$, the corresponding right moving frame is obtained by solving the normalization equations

$$
\begin{gathered}
X^{i_{1}}\left(z^{(n)}, g^{(n)}\right)=c_{1}, \ldots, X^{i_{l}}\left(z^{(n)}, g^{(n)}\right)=c_{l}, \\
U_{X^{J_{l+1}}}^{\alpha_{l+1}}\left(z^{(n)}, g^{(n)}\right)=c_{l+1}, \ldots, U_{X^{J_{d_{n}}}}^{\alpha_{d_{n}}}\left(z^{(n)}, g^{(n)}\right)=c_{d_{n}},
\end{gathered}
$$

for the pseudogroup jets $g^{(n)}=\rho^{(n)}\left(z^{(n)}\right)$ so that

$$
\varrho^{(n)}\left(z^{(n)}\right)=\left(z^{(n)}, \rho^{(n)}\left(z^{(n)}\right)\right) .
$$

To each right moving frame (19) corresponds a unique left moving frame $\bar{\varrho}^{(n)}$ obtained by pseudogroup inversion:

$$
\bar{\varrho}^{(n)}\left(\rho^{(n)}\left(z^{(n)}\right) \cdot z^{(n)}\right)=\left(\rho^{(n)}\left(z^{(n)}\right) \cdot z^{(n)},\left(\rho^{(n)}\left(z^{(n)}\right)\right)^{-1}\right) .
$$


In the following, we let $\bar{\rho}^{(n)}\left(z^{(n)}\right)=\left(\rho^{(n)}\left(z^{(n)}\right)\right)^{-1}$ denote the inverse of the pseudogroup jet $\rho^{(n)}\left(z^{(n)}\right)$ so that

$$
\bar{\varrho}^{(n)}\left(z^{(n)}\right)=\left(\rho^{(n)}\left(z^{(n)}\right) \cdot z^{(n)}, \bar{\rho}^{(n)}\left(z^{(n)}\right)\right) \quad \text { where } z^{(n)}=\boldsymbol{\tau}^{(n)}\left(\bar{\varrho}^{(n)}\right) .
$$

Given a (right) moving frame, there is a systematic procedure for invariantizing differential functions and differential forms. First recall the standard coframe on $\mathbf{J}^{\infty}$ given by the horizontal one-forms

$$
d x^{1}, \ldots, d x^{p}
$$

and the basic contact one-forms

$$
\theta_{J}^{\alpha}=d u_{J}^{\alpha}-\sum_{j=1}^{p} u_{J, j}^{\alpha} d x^{j}, \quad \alpha=1, \ldots, q, \# J \geqslant 0 .
$$

Supplementing (21) with the Maurer-Cartan forms $\left.\mu_{A}^{a}\right|_{\mathcal{G}}$ yields a coframe for the lifted bundle $\mathcal{E}^{(\infty)}$.

DEFinition 10. Let $\omega$ be a differential form on $\mathrm{J}^{\infty}$. Its lift is the $\mathcal{G}$-invariant differential form

$$
\lambda(\omega)=\pi_{\mathrm{J}}\left[\tau^{*}(\omega)\right]
$$

where $\pi_{\mathrm{J}}$ is the projection onto jet forms obtained by setting the Maurer-Cartan forms equal to zero.

We denote by

$$
\Omega^{i}=\lambda\left(d x^{i}\right), \quad \Theta_{J}^{\alpha}=\lambda\left(\theta_{J}^{\alpha}\right),
$$

the lift of the standard jet coframe. When $\omega$ is a submanifold jet coordinate, its lift is just the usual prolonged action:

$$
X^{i}=\lambda\left(x^{i}\right), \quad U_{X^{J}}^{\alpha}=\lambda\left(u_{J}^{\alpha}\right) .
$$

The lift map (22) may also be extended to the vector field jet $\zeta^{(n)}$ by defining

$$
\lambda\left(\zeta_{A}^{a}\right)=\mu_{A}^{a}
$$

to be the corresponding right-invariant Maurer-Cartan form.

Definition 11. Let $\varrho=\varrho^{(\infty)}: \mathrm{J}^{\infty} \rightarrow \mathcal{E}^{(\infty)}$ be a right moving frame. Then the invariantization map $\iota: \boldsymbol{\Omega}^{*}\left(\mathbf{J}^{\infty}\right) \rightarrow \boldsymbol{\Omega}^{*}\left(\mathbf{J}^{\infty}\right)$ is defined by

$$
\iota=\varrho^{*} \circ \lambda \text {. }
$$


We denote by

$$
\begin{aligned}
& \varpi^{i}=\varrho^{*}\left(\Omega^{i}\right)=\iota\left(d x^{i}\right), \quad i=1, \ldots, p, \\
& \vartheta_{J}^{\alpha}=\varrho^{*}\left(\Theta_{J}^{\alpha}\right)=\iota\left(\theta_{J}^{\alpha}\right), \quad \alpha=1, \ldots, q, \# J \geqslant 0,
\end{aligned}
$$

the invariantization of the horizontal coframe and basic contact one-forms. Since the lifted contact forms (23) and their invariant counterparts in (25) are not used in the group foliation method, we introduce the equivalence notation $\equiv$ to denote equality of two differential one-forms up to a lifted or invariant contact form. Finally, we denote the invariantization of the submanifold jet coordinates by

$$
H^{i}=\iota\left(x^{i}\right), \quad I_{J}^{\alpha}=\iota\left(u_{J}^{\alpha}\right),
$$

and refer to them as normalized invariants. By construction, the invariantization of the jet coordinates (18) defining the cross-section $\mathcal{K}^{\infty}$ are constant, and for this reason they are called phantom invariants.

Proposition 12. The normalized invariants (26) form a complete set of functionally independent differential invariants. In particular, using the invariantization map (24), any invariant $J\left(x, u^{(n)}\right)$ can be expressed as

$$
J\left(x, u^{(n)}\right)=\iota\left[J\left(x, u^{(n)}\right)\right]=J\left(H, I^{(n)}\right) .
$$

EXAMPLE 13. We now construct a moving frame for the Lie pseudogroup action (3). A standard cross-section to the pseudogroup orbits is

$$
x=0, \quad u=1, \quad u_{x^{k}}=0, \quad k \geqslant 1 .
$$

Solving the normalization equations $U=1, X=U_{X^{k}}=0, k \geqslant 1$, for the pseudogroup parameters yields the right moving frame

$$
f=0, \quad f_{x^{k}}=u_{x^{k-1}}, \quad k \geqslant 1 .
$$

Up to second order, the invariantization of the submanifold jet coordinates produces the normalized invariants

$$
\begin{gathered}
H^{y}=\iota(y)=y, \quad I_{01}=\iota\left(u_{y}\right)=\frac{u_{y}}{u}, \\
I_{11}=\iota\left(u_{x y}\right)=\frac{u u_{x y}-u_{x} u_{y}}{u^{3}}, \quad I_{02}=\iota\left(u_{y y}\right)=\frac{u_{y y}}{u} .
\end{gathered}
$$

The invariantization of the horizontal coframe gives the invariant one-forms

$$
\varpi^{x}=\varrho^{*}\left(d_{J} X\right)=u d x, \quad \varpi^{y}=\varrho^{*}\left(d_{J} Y\right)=d y .
$$


The corresponding left moving frame is obtained by inverting the right moving frame (28):

$$
\bar{f}=x, \quad \bar{f}_{X}=\frac{1}{f_{x}}=\frac{1}{u}, \quad \bar{f}_{X X}=-\frac{f_{x x}}{f_{x}^{3}}=\frac{u_{x}}{u^{3}}, \quad \ldots
$$

THEOREM 14. Let $\omega$ be a differential form on $\mathrm{J}^{\infty}$. Then

$$
d[\lambda(\omega)]=\lambda\left[d \omega+\mathbf{v}^{(\infty)}(\omega)\right] .
$$

An immediate consequence of (31) is that

$$
d[\iota(\omega)]=\iota\left[d \omega+\mathbf{v}^{(\infty)}(\omega)\right] .
$$

The identity (32) is called the universal recurrence relation. We are particularly interested in the case when $\omega$ is a differential function, and, more particularly, when $\omega$ is one of the submanifold jet coordinates. Substituting $x^{i}$ and $u_{J}^{\alpha}$ in (32), we obtain the invariant recurrence relations

$$
d H^{i}=\varpi^{i}+M^{i}, \quad d I_{J}^{\alpha} \equiv I_{J, i}^{\alpha} \varpi^{i}+N_{J}^{\alpha},
$$

for the normalized invariants. The correction terms $M^{i}, N_{J}^{\alpha}$ come from the Lie algebraic term $\iota\left(\mathbf{v}^{(\infty)}(\omega)\right)$ in (32). One of most important features of (33) or (32) is that these equations do not require the coordinate expression of the invariant object to be computed [43].

EXAMPLE 15. In this example, we compute the invariant recurrence relations (33) for the normalized invariants (29). To compute the lifted recurrence relation (31), we need the prolongation of the infinitesimal generator (7):

$$
\begin{aligned}
\mathbf{v}^{(\infty)}= & a(x) \frac{\partial}{\partial x}-u a_{x} \frac{\partial}{\partial u}-\left(u a_{x x}+2 u_{x} a_{x}\right) \frac{\partial}{\partial u_{x}}-u_{y} a_{x} \frac{\partial}{\partial u_{y}}-u_{y y} a_{x} \frac{\partial}{\partial u_{y y}} \\
& -\left(u_{y} a_{x x}+2 u_{x y} a_{x}\right) \frac{\partial}{\partial u_{x y}}-\left(u a_{x x x}+3 u_{x} a_{x x}+3 u_{x x} a_{x}\right) \frac{\partial}{\partial u_{x x}}-\cdots .
\end{aligned}
$$

Substituting $x, y, u, u_{x}, u_{y}, \ldots$ for $\omega$ in (31), we obtain, modulo contact forms,

$$
\begin{aligned}
& d X=\Omega^{x}+\mu, \quad d Y=\Omega^{y}, \\
& d U \equiv U_{X} \Omega^{x}+U_{Y} \Omega^{y}-U \mu_{X}, \\
& d U_{X} \equiv U_{X X} \Omega^{x}+U_{X Y} \Omega^{y}-U \mu_{X X}-2 U_{X} \mu_{X}, \\
& d U_{Y} \equiv U_{X Y} \Omega^{x}+U_{Y Y} \Omega^{y}-U_{Y} \mu_{X}, \\
& d U_{X X} \equiv U_{X X X} \Omega^{x}+U_{X X Y} \Omega^{y}-U \mu_{X X X}-3 U_{X} \mu_{X X}-3 U_{X X} \mu_{X}, \\
& d U_{X Y} \equiv U_{X X Y} \Omega^{x}+U_{X Y Y} \Omega^{y}-U_{Y} \mu_{X X}-2 U_{X Y} \mu_{X}, \\
& d U_{Y Y} \equiv U_{X Y Y} \Omega^{x}+U_{Y Y Y} \Omega^{y}-U_{Y Y} \mu_{X}, \quad \ldots
\end{aligned}
$$


Pulling back (34) by the right moving frame $\rho$, the left-hand side of (34) is identically zero for the phantom invariants $H^{x}=0, I=1, I_{k 0}=0, k \geqslant 1$. Solving these equations for the pulled-back Maurer-Cartan forms $\mu_{k}=\varrho^{*} \mu_{k}$, the result is

$$
\mu=-\varpi^{x}, \quad \mu_{k} \equiv I_{k-1,1} \varpi^{y}, \quad k \geqslant 1 .
$$

Substituting the expressions (35) into the remaining recurrence relations (34) yields the invariant recurrence relations

$$
\begin{gathered}
d H^{y}=\varpi^{y}, \quad d I_{01} \equiv I_{11} \varpi^{x}+I_{02} \varpi^{y}-I_{01}^{2} \varpi^{y}, \\
d I_{11} \equiv I_{21} \varpi^{x}+I_{12} \varpi^{y}-3 I_{01} I_{11} \varpi^{y}, \quad d I_{02} \equiv I_{12} \varpi^{x}+I_{03} \varpi^{y}-I_{01} I_{02} \varpi^{y},
\end{gathered}
$$

and so on. Let $\mathcal{D}_{i}$ be the invariant total differential operators dual to the invariant horizontal one-forms $\varpi^{i}$ defined by

$$
d F \equiv \sum_{i=1}^{p} \mathcal{D}_{i}(F) \varpi^{i} \quad \text { for any differential function } F\left(x, u^{(n)}\right) .
$$

Since the invariant horizontal one-forms $\varpi^{i}$ are linearly independent, we deduce from (36) the recurrence relations

$$
\begin{array}{ll}
\mathcal{D}_{x} I_{01}=I_{11}, & \mathcal{D}_{y} I_{01}=I_{02}-I_{01}^{2}, \\
\mathcal{D}_{x} I_{11}=I_{21}, & \mathcal{D}_{y} I_{11}=I_{12}-3 I_{01} I_{11}, \\
\mathcal{D}_{x} I_{02}=I_{12}, & \mathcal{D}_{y} I_{02}=I_{03}-I_{01} I_{02},
\end{array}
$$

among the low-order normalized invariants. Note that the invariant $I_{12}$ appears twice on the right-hand side of (37). Eliminating this invariant, we obtain the relation

$$
\mathcal{D}_{x} I_{02}=\mathcal{D}_{y} I_{11}+3 I_{01} I_{11}
$$

DEFINITION 16. A set of invariants $\mathcal{I}$ is said to generate the algebra of differential invariants with respect to the invariant derivative operators $\mathcal{D}_{1}, \ldots$, $\mathcal{D}_{p}$ if all differential invariants can be expressed as some function of the invariants $I \in \mathcal{I}$ and their invariant derivatives $\mathcal{D}_{J} I$.

The fundamental basis theorem-first proved for finite-dimensional group actions by Lie [26, page 760], and later extended to infinite-dimensional Lie pseudogroups by Tresse [54] - guarantees that the set $\mathcal{I}$ may be taken to be finite. That is, the algebra of differential invariants is generated by a finite number of invariants. Modern proofs of the fundamental basis theorem appear in the textbooks [36, 47]; other proofs based on Spencer cohomology [23], Weyl algebras [32], homological methods [21], or moving frames [15, 39, 44] also exist. 
Using Gröbner basis techniques, the proof of the basis theorem presented in [44] is constructive and also identifies a generating set. The proof relies on the assumption that moving frames constructed are of minimal order, and hence we assume from now on every moving frame to be of minimal order. Intuitively, a moving frame is of minimal order if during the normalization procedure the pseudogroup parameters are normalized as soon as possible; we refer the reader to $[15,39]$ for a precise definition.

Understanding functional dependence relations among the invariants will be central to the implementation of the group foliation method.

DEFINITION 17. A syzygy among the generating differential invariants

$$
\mathcal{I}=\left\{I^{1}, \ldots, I^{k}\right\}
$$

is a nontrivial functional relationship

$$
S\left(\ldots, \mathcal{D}_{L} I^{1}, \ldots, \mathcal{D}_{K} I^{k}, \ldots\right)=0
$$

among the invariants $I^{v}$ and their various invariant derivatives $\mathcal{D}_{J} I^{\nu}$.

EXAmple 18. Continuing Example 15, setting $\omega=d x$ and $\omega=d y$ in the recurrence relation (32), we find that

$$
d \varpi^{x}=I_{01} \varpi^{y} \wedge \varpi^{x}, \quad d \varpi^{y}=0 .
$$

By duality, we deduce the commutation relation

$$
\left[\mathcal{D}_{x}, \mathcal{D}_{y}\right]=I_{01} \mathcal{D}_{x}
$$

Syzygies arising from commutation relations such as the above are called commutator syzygies. For example, for any differential invariant $I$, one finds by application of (39) the syzygy

$$
\mathcal{D}_{x} \mathcal{D}_{y} I=\mathcal{D}_{y} \mathcal{D}_{x} I+I_{01} \mathcal{D}_{x} I .
$$

Definition 19. A collection $\mathcal{S}=\left\{S_{1}, \ldots, S_{k}\right\}$ of syzygies is said to form a generating system if every syzygy can be written as a linear combination of members of $\mathcal{S}$ and finitely many of their derivatives, modulo the commutator syzygies.

THEOREM 20. Let $\mathcal{G}$ be a Lie pseudogroup acting locally freely on an open subset of the submanifold jet bundle $\mathbf{J}^{n}$ for some $n \geqslant 1$. Then the algebra of syzygies is generated by a finite number of fundamental syzygies. 
A comprehensive discussion of syzygies and a proof of Theorem 20 appears in [44]. The proof is again constructive and based on Gröbner basis methods. We note that in applications it is generally possible to avoid the introduction of the Gröbner basis machinery. The generating sets $\mathcal{I}$ and $\mathcal{S}$ for the algebra of differential invariants and the algebra of syzygies can be found by direct observation.

EXAMPLE 21. Continuing Example 15, we conclude from the recurrence relations (37) that the second-order normalized invariants $I_{11}$ and $I_{02}$ are expressible in terms of the normalized invariants $I_{01}$ and $H^{y}$ and their invariant derivatives with respect to $\mathcal{D}_{x}$ and $\mathcal{D}_{y}$. The same holds for higher-order normalized invariants, and we conclude that the algebra of differential invariants of the pseudogroup (3) is generated by the normalized invariants $I_{01}$ and $H^{y}$ and the invariant derivative operators $\mathcal{D}_{x}=(1 / u) D_{x}$ and $\mathcal{D}_{y}=D_{y}$.

Also, there is no fundamental syzygy among the generating invariants $\left\{H^{y}, I_{01}\right\}$. Every syzygy must be trivial modulo the commutator syzygies. For example, substituting $I=I_{01}$ in (40) and using the recurrence relations (37), we recover the syzygy (38).

In the above discussion, the invariant derivative operators $\mathcal{D}_{1}, \ldots, \mathcal{D}_{p}$ can be replaced by any other set of $p$ linearly independent invariant total derivative operators. In doing so, the structure of the algebra of differential invariants may change. As the next example shows, the generating set of invariants and fundamental syzygies are dependent on the basis of invariant total derivative operators.

EXAMPLE 22. We now revisit Example 21, using a different set of invariant derivative operators. To simplify the notation, let

$$
H=H^{y}, \quad J=I_{01}, \quad K=I_{11}, \quad L=I_{02} .
$$

From (36), we have that

$$
d H \wedge d J \equiv K \varpi^{y} \wedge \varpi^{x} .
$$

Working on the open subset of jet space where $K \neq 0$, one can replace the invariant total derivative operators $\mathcal{D}_{x}$ and $\mathcal{D}_{y}$ by the invariant Tresse derivatives $D_{H}$ and $D_{J}[22]$. By the chain rule,

$$
\begin{aligned}
& \mathcal{D}_{x}=\mathcal{D}_{x} H \cdot D_{H}+\mathcal{D}_{x} J \cdot D_{J}=K D_{J}, \\
& \mathcal{D}_{y}=\mathcal{D}_{y} H \cdot D_{H}+\mathcal{D}_{y} J \cdot D_{J}=D_{H}+\left(L-J^{2}\right) D_{J} .
\end{aligned}
$$


In terms of these Tresse derivatives, the algebra of differential invariants cannot be generated by the invariants $\left\{H^{y}, I_{01}\right\}=\{H, J\}$, since

$$
D_{H}(H)=D_{J}(J)=1 \quad \text { and } \quad D_{H}(J)=D_{J}(H)=0 .
$$

In this case, a generating set of invariants is given by the four normalized invariants (41). There is now one fundamental syzygy obtained by expressing (38) in terms of the operators (43):

$$
\mathcal{D}_{x} L=\mathcal{D}_{y} K+3 J K \quad \Longleftrightarrow \quad K D_{J} L=D_{H} K+\left(L-J^{2}\right) D_{J} K+3 J K .
$$

\section{Group foliation}

In the first part of this section, we review the classical method of group foliation, mostly following Ovsiannikov's treatment [47]. Moving frames are used when possible to simplify the constructions. In particular, the derivation of the automorphic and resolving systems is done symbolically without relying on coordinate expressions for the differential invariants. In the second part of this section, the moving frame method is used to obtain a symbolic procedure for reconstructing solutions of the original differential equation from solutions of the resolving system. This reconstruction procedure differs from the classical approach using automorphic systems, which requires explicit formulas for differential invariants.

4.1. Vessiot's group foliation method. Given a differential equation $\Delta=0$, group foliation splits the problem of solving $\Delta=0$ into one of solving two associated systems of differential equations called the resolving and automorphic systems. More precisely, it is an associated family of equations; each solution to the resolving system determines a particular $\mathcal{G}$-automorphic system, which in turns yields solutions to the original equation $\Delta=0$.

DEFINITION 23. A system of differential equations is called $\mathcal{G}$-automorphic if all of its solutions can be obtained from a single solution via transformations belonging to $\mathcal{G}$.

We now describe the method rigorously. Suppose that

$$
\Delta\left(x, u^{(n)}\right)=0
$$

is an $n$ th-order differential equation admitting a Lie pseudogroup $\mathcal{G}$ of symmetries. By definition of invariance, $\mathcal{G}$ maps solutions of $\Delta=0$ to other 


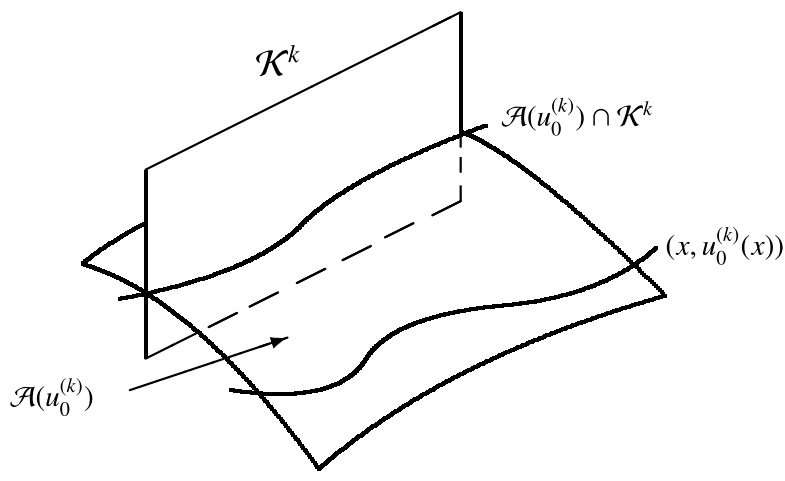

Figure 2. The orbit of a graph and intersection with a cross-section.

solutions. Thus, there is an induced action of $\mathcal{G}$ on the solution set, partitioning this space into orbits. If the jets of solutions lie within the set of regular jets $\mathcal{V}^{k} \subset \mathbf{J}^{k}$, these orbits determine invariant submanifolds in $\mathrm{J}^{k}, k \geqslant 0$, traced out by the action of $\mathcal{G}$ on the prolonged graph of a given solution. The description of these invariant submanifolds using the differential invariants of $\mathcal{G}$ leads to the main idea of the group foliation method.

Let $\mathcal{K}^{k}$ be a cross-section to the prolonged action of $\mathcal{G}$ on $\mathrm{J}^{k}$, and let $u_{0}: X \rightarrow U$ be an arbitrary function whose prolonged graph $\left(x, u_{0}^{(k)}(x)\right)$ lies in a neighborhood of $\mathcal{K}^{k}$. Later on, $u_{0}(x)$ will be a solution of the differential equation $\Delta=0$, but the immediate discussion does not rely on this assumption. Consider the orbit under $\mathcal{G}$ of the $k$ th prolongation of the graph $\left(x, u_{0}^{(k)}(x)\right)$ :

$$
\mathcal{A}\left(u_{0}^{(k)}\right)=\left\{g^{(k)} \cdot\left(x, u_{0}^{(k)}(x)\right):\left.g^{(k)} \in \mathcal{G}^{(k)}\right|_{\left(x, u_{0}^{(k)}\right)}\right\} \subset \mathbf{J}^{k} .
$$

Let $r_{k}$ be the dimension of the intersection of $\mathcal{A}\left(u_{0}^{(k)}\right)$ with the cross-section $\mathcal{K}^{k}$. We assume that the dimension of this intersection is constant. Increasing the order of prolongation, we have the nondecreasing sequence

$$
0 \leqslant r_{0} \leqslant r_{1} \leqslant \cdots \leqslant p
$$

DEFinition 24. The smallest order $s$ such that $r_{s}=r_{s+i}$ for all $i \geqslant 1$ is called the order, and $r=r_{s}$ is called the invariant rank of the function $u_{0}$.

As guaranteed by the fundamental basis theorem, we may choose $k \geqslant s$ so that there is a functionally independent generating set $\mathcal{I}$ of differential invariants of order $\leqslant k$. These invariants provide coordinates for the cross-section $\mathcal{K}^{k}$, and this 
allows us to write the intersection $\mathcal{A}\left(u_{0}^{(k)}\right) \cap \mathcal{K}^{k}$ as a parameterized submanifold of $\mathcal{K}^{k}$. For this purpose, distinguish a set of functionally independent differential invariants $\left\{J^{1}, \ldots, J^{r}\right\} \subset \mathcal{I}$, to be used as parametric variables. We may then use the remaining invariants as dependent variables for the parameterization, writing $\mathcal{A}\left(u_{0}^{(k)}\right) \cap \mathcal{K}^{k}$ locally as a graph in $\mathcal{K}^{k}$ :

$$
\mathcal{A}_{r}: K^{1}=F^{1}\left(J^{1}, \ldots, J^{r}\right), \ldots, K^{v}=F^{v}\left(J^{1}, \ldots, J^{r}\right),
$$

where $\left\{J^{1}, \ldots, J^{r}, K^{1}, \ldots, K^{\nu}\right\}$ is the full generating set of invariants. The system (45) is automorphic, and it will be called an automorphic system $\mathcal{A}_{r}$ of rank $r$, dropping reference to $u_{0}$. In [47], it is shown that every automorphic system on $\mathbf{J}^{\infty}$ has the form (45).

REMARK 25. In practice, we may distinguish the invariants $J^{1}, \ldots, J^{r}$ by verifying the independence condition

$$
d J^{1} \wedge \cdots \wedge d J^{r} \not \equiv 0
$$

on $\mathcal{A}_{r}$. This may be done symbolically, without the need for explicit formulas for the invariants.

EXAMPLE 26. In this example, we obtain the automorphic systems for the pseudogroup (3). The differential invariants and their recurrence relations were obtained in Examples 13 and 15. Since the independent variable $H=H^{y}=y$ is an invariant, the invariant rank of an automorphic system is bounded by $1 \leqslant r \leqslant 2$. Distinguishing the invariants $H$ and $J$ as parameters, the independence condition (42) from Example 22 implies that, when $K \neq 0$, the invariants $H, J$ are independent, and automorphic systems of rank two have the form

$$
\mathcal{A}_{2}:\left\{\begin{array} { l } 
{ K = F ^ { 1 } ( H , J ) } \\
{ L = F ^ { 2 } ( H , J ) }
\end{array} \Rightarrow \left\{\begin{array}{l}
\frac{u u_{x y}-u_{x} u_{y}}{u^{3}}=F^{1}\left(y, \frac{u_{y}}{u}\right) \\
\frac{u_{y y}}{u}=F^{2}\left(y, \frac{u_{y}}{u}\right) .
\end{array}\right.\right.
$$

When $K=0$, we may choose $H$ as a parameter to obtain the rank-one automorphic systems

$$
\mathcal{A}_{1}:\left\{\begin{array} { l } 
{ J = F ^ { 1 } ( H ) } \\
{ K = 0 } \\
{ L = F ^ { 2 } ( H ) }
\end{array} \Rightarrow \left\{\begin{array}{l}
\frac{u_{y}}{u}=F^{1}(y) \\
\frac{u u_{x y}-u_{x} u_{y}}{u^{3}}=0 \\
\frac{u_{y y}}{u}=F^{2}(y) .
\end{array}\right.\right.
$$


The choice of $F^{1}, \ldots, F^{v}$ in (45) may not be arbitrary. Because (45) is expressed in terms of differential invariants, applications of syzygies among the invariants will lead to integrability conditions. Consideration of these syzygies leads to a system of differential equations for the functions $F^{1}, \ldots, F^{v}$ in the automorphic system $\mathcal{A}_{r}$ that we call the syzygy system.

We first discuss syzygy systems for full rank automorphic systems, that is $r=p$. Let $\mathcal{S}$ be the set of fundamental syzygies among the generating invariants $\mathcal{I}=\left\{J^{1}, \ldots, J^{r}, K^{1}, \ldots, K^{\nu}\right\}$. Making the chain rule substitutions

$$
\mathcal{D}_{i}=\sum_{k=1}^{r}\left(\mathcal{D}_{i} J^{k}\right) D_{J^{k}}, \quad i=1, \ldots, p,
$$

we may write the invariant differential operators $\mathcal{D}_{i}$ appearing in each syzygy in terms of the derivatives $D_{J^{j}}$. Without loss of generality, we assume that $\mathcal{D}_{i} J^{j}$, $i=1, \ldots, p, j=1, \ldots, r$, are again functions of the generating invariants $\mathcal{I}$ by increasing the order of prolongation and adding more invariants to $\mathcal{I}$ if necessary (we do not require $\mathcal{I}$ to be minimal). Application of the fundamental syzygies to the system (45) results in a system of differential equations for $F^{1}, \ldots, F^{v}$. This system is called the syzygy system.

REMARK 27. As can be seen from Example 22, for any particular symmetry group, the substitution (48) may be made symbolically, without explicit formulas for the invariants, using the recurrence relations (33).

EXAMPLE 28. The syzygy system associated to the rank-two automorphic system (46) is simply obtained by substituting the functions $K=F^{1}(H, J)$ and $L=F^{2}(H, J)$ into the fundamental syzygy (44), resulting in the first-order partial differential equation

$$
F^{1} \frac{\partial F^{2}}{\partial J}=\frac{\partial F^{1}}{\partial H}+\left(F^{2}-J^{2}\right) \frac{\partial F^{1}}{\partial J}+3 J F^{1} .
$$

We now address the case when the automorphic systems considered have less than full rank, that is $r<p$. In this instance, the substitution (48) may introduce new dependencies among the differentiated invariants in addition to the fundamental syzygies and their consequences. We will call these dependencies restriction syzygies, since they arise from restricting the differential operators to submanifolds (locally) parameterized by $J^{1}, \ldots, J^{r}$.

EXAMPLE 29. For the rank-one automorphic system (47), the syzygy (44) is trivial. To see this, express the invariant total derivative operators $\mathcal{D}_{x}, \mathcal{D}_{y}$ in terms 
of the single operator $D_{H}$ :

$$
\mathcal{D}_{x}=\mathcal{D}_{x} H \cdot D_{H}=0, \quad \mathcal{D}_{y}=\mathcal{D}_{y} H \cdot D_{H}=D_{H} .
$$

On the other hand, by substitution of (50) into the recurrence relations (37), we find

$$
D_{H} J=L-J^{2}, \quad I_{21}=0, \quad I_{12}=0, \quad I_{03}=D_{H} L-J L,
$$

and so on. Thus, there is a new restriction syzygy

$$
D_{H} J=L-J^{2}
$$

among the generating invariants, arising from the restriction of the invariants and invariant differential operators to submanifolds of the form (47). It can be seen by inspection that this restriction syzygy is generating. Thus we arrive at the rank-one syzygy system for the functions $F^{1}(H), F^{2}(H)$ :

$$
\frac{\partial F^{1}}{\partial H}=F^{2}-\left(F^{1}\right)^{2} .
$$

REMARK 30. We will henceforth refrain from referencing the functions $F^{i}$ in our examples when it is understood that each invariant $K^{i}$ is a function $K^{i}\left(J^{1}, \ldots, J^{r}\right)$.

Analogous to Theorem 20 in the full rank case $r=p$ (where the restriction syzygies are identical to the usual syzygies), the restriction syzygies for $r<p$ are also finitely generated.

PROPOSITION 31. Suppose that the Lie pseudogroup $\mathcal{G}$ admits a moving frame. For any choice of distinguished invariants $J^{1}, \ldots, J^{r}$, the set of restriction syzygies resulting from substitution of the relations (48) into the recurrence relations is finitely generated. A finite generating set of restriction syzygies is called a set of fundamental restriction syzygies.

Proof. The full rank case $r=p$ follows from Theorem 20. When $r<p$, certain constraints among the differential invariants are imposed, as can be seen in Example 26. Writing the differential invariants explicitly in terms of submanifold jet coordinates $\left(x, u^{(n)}\right)$, these constraints give invariant differential equations that $u=u(x)$ must satisfy. The proposition then follows from the fact that the differential module of differential syzygies restricted to the solution space of an invariant differential equation is finitely generated [22, Theorem 24]. 
REMARK 32. Our proof of Proposition 31 provides only the existence of a finite generating set of restriction syzygies. A constructive proof would be preferable and useful for more intensive examples than those treated in this paper.

We are now prepared to define the syzygy system for all ranks $r \leqslant p$.

DEFINITION 33. The syzygy system $\mathcal{S}_{r}$ for a rank-r automorphic system $\mathcal{A}_{r}$ is the finite system of differential equations for $F^{1}, \ldots, F^{v}$ as functions of the invariant parameters $J^{1}, \ldots, J^{r}$ obtained by applying to $\mathcal{A}_{r}$ the fundamental restriction syzygies.

REMARK 34. It is important to note that the syzygy system does not impose extra conditions on the solutions of the system $\mathcal{A}_{r} ; \mathcal{S}_{r}$ is a collection of integrability conditions on the functions $F^{j}$.

Let us now return to the context in which our automorphic systems (45) arise as orbits of solutions $u_{0}(x)$ to a $\mathcal{G}$-invariant differential equation $\Delta=0$, and discuss how to apply these systems to the problem of finding solutions to $\Delta=0$.

Starting with a solution $u_{0}(x)$ to a $\mathcal{G}$-invariant equation $\Delta=0$, solutions to the $\mathcal{G}$-automorphic system $\mathcal{A}\left(u_{0}^{(k)}\right)$ will again satisfy $\Delta=0$ by invariance. Unfortunately, this observation does not offer obvious practical value for finding solutions to $\Delta=0$; indeed, if a 'seed' solution $u_{0}$ is known, one can simply apply the pseudogroup transformations to $u_{0}$ and avoid automorphic systems altogether. The preceding construction of syzygy systems suggests an alternative approach: append to the syzygy system the condition $\Delta=0$. By adding this condition, we ensure that the automorphic systems determined by solving the syzygy system are those generated by solutions to $\Delta=0$. Note that, by the invariance of $\Delta=0$, this amounts to adding new relations among the generating invariants; these relations will be called constraint syzygies. The constraint syzygies together with the restriction syzygies give a set of differential equations, called the resolving system, whose solutions determine automorphic systems generated by solutions of $\Delta=0$.

DEFINITION 35. The rank-r resolving system $\mathcal{R}_{r}(\Delta)$ of a differential equation $\Delta=0$ foliated by $\mathcal{G}$ is the system of differential equations obtained by appending to the syzygy system $\mathcal{S}_{r}$ the constraint syzygy $\iota(\Delta)=0$ and its differential consequences.

EXAMPLE 36. We now obtain the rank-two resolving system for the nonlinear wave equation

$$
u u_{x y}-u_{x} u_{y}=u^{3}
$$


foliated by the Lie pseudogroup (3). First observe that this Lie pseudogroup is a symmetry group of (52). Invariantization of (52) gives the constraint syzygy

$$
K=1 \text {. }
$$

Appending the constraint syzygy to the syzygy system (49) yields the resolving system

$$
K=1, \quad D_{J} L=3 J .
$$

Note that there is no rank-one resolving system, because the constraint syzygy (53) is not compatible with the dependence condition $K=0$ from (42).

REMARK 37. The addition of the constraint syzygy may, as usual, be performed symbolically by direct invariantization of the equation $\Delta=0$ and use of the recurrence relation to write all invariants appearing in $\iota(\Delta)$ in terms of the generating invariants. We assume that the solution space of $\Delta=0$ lies within the set of regular jets so that the equation may be written as a level set of differential invariants; see [35, Proposition 2.56].

All the ingredients for the group foliation algorithm are now in place.

Algorithm 38 (Group foliation). Let $\Delta\left(x, u^{(n)}\right)=0$ be an $n$ th-order differential equation invariant under a Lie pseudogroup $\mathcal{G}$, and suppose that $\mathcal{G}$ admits a moving frame on the solution space of $\Delta=0$.

- Choose an invariant rank $r$ for which rank-r solutions will be sought. Prolong to order $k \geqslant s$, where $s$ is the order of stabilization of a generic rank-r solution, so that the normalized invariants of order at most $k$ form a generating set.

- Choose distinguished invariants $J^{1}, \ldots, J^{r}$ among the normalized invariants so that $\mathcal{D}_{i} J^{j}$ have order no greater than $k$. These invariants will be used as independent variables and the remaining normalized invariants $K^{1}, \ldots, K^{v}$ as dependent variables in the automorphic system

$$
\mathcal{A}_{r}: K^{1}=F^{1}\left(J^{1}, \ldots, J^{r}\right), \ldots, K^{\nu}=F^{\nu}\left(J^{1}, \ldots, J^{r}\right) .
$$

- Compute the order- $r$ resolving system $\mathcal{R}_{r}(\Delta)$ by applying the restriction syzygies and the constraint syzygy $\iota(\Delta)=0$ to $\mathcal{A}_{r}$.

- Find a solution $F^{1}\left(J^{1}, \ldots, J^{r}\right), \ldots, F^{v}\left(J^{1}, \ldots, J^{r}\right)$ to the resolving system.

- Form an automorphic system $\mathcal{A}_{r}$ using the resolving system solution, and write the invariants in this automorphic system explicitly in terms of $\left(x, u^{(k)}\right)$. Solutions of this automorphic system will satisfy the original equation $\Delta=0$. 
EXAMPLE 39. We continue Example 36. A general solution to the resolving system (54) is easily found:

$$
K(H, J)=1, \quad L(H, J)=\frac{3}{2} J^{2}+G(H),
$$

where $G(H)$ is an arbitrary smooth function. Substituting (55) and the explicit formulas (29) for the invariants into the automorphic system (46), we obtain the system of differential equations

$$
\frac{u u_{x y}-u_{x} u_{y}}{u^{3}}=1, \quad \frac{u_{y y}}{u}=\frac{3}{2}\left(\frac{u_{y}}{u}\right)^{2}+G(y) .
$$

It is apparent that the method in this instance has been circular; the original equation itself appears in the final automorphic system, and the second equation of (56) follows from the first by cross-differentiation. This unfortunate outcome will be remedied by the subject of the next section.

EXAMPLE 40. To illustrate the algorithm for nonmaximal invariant rank, we consider the differential equation

$$
u u_{x y}-u_{x} u_{y}=0 .
$$

This equation also admits the symmetry pseudogroup (3). Using the same notation as Examples 26 and 29, (57) implies the constraint syzygy $K=0$. Since $d H \wedge$ $d J \equiv K \varpi^{y} \wedge \varpi^{x}=0$, the invariants $H$ and $J$ are functionally dependent, and the resolving equations in this case are identical to the rank-one syzygy system already computed in Example 29. A solution to the resolving system is

$$
J(H)=G(H), \quad L(H)=G^{\prime}(H)+G(H)^{2},
$$

where $G$ is an arbitrary smooth function. Substituting (58) and the explicit formulas (29) for the invariants into the automorphic system (47), we obtain the system of differential equations

$$
\frac{u_{y}}{u}=G(y), \quad \frac{u u_{x y}-u_{x} u_{y}}{u^{3}}=0, \quad \frac{u_{y y}}{u}=G^{\prime}(y)+G(y)^{2} .
$$

We do not pursue a solution of (59) at present. This will be done by alternative means in Example 49 to follow.

In Algorithm 38, all steps except for the last may be executed using the symbolic calculus of moving frames. It is only the last step that requires explicit knowledge of the differential invariants and, in the instance of Example 39, leads to a dead end in the computation. In keeping with the intent of moving frames, we propose an alternative method for reconstruction of solutions from the resolving system that is completely symbolic, and effective in certain examples, such as Example 39, where the standard reconstruction method fails. 


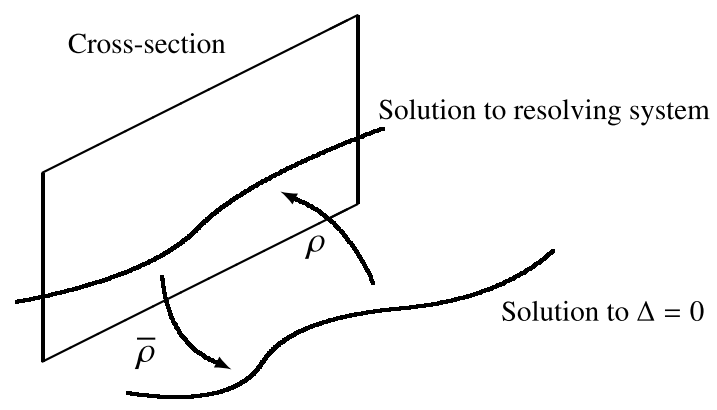

Figure 3. The geometry of reconstruction.

4.2. Reconstruction procedure. The method of moving frames is naturally incorporated into our exposition of the group foliation method. Moving frames are not required per se to perform the algorithm, but they facilitate the symbolic construction of the automorphic and resolving systems using only the infinitesimal data of the pseudogroup action and the choice of a cross-section to the pseudogroup orbits. But, when the automorphic system is used to construct a solution to $\Delta=0$ from a solution of the resolving system, as in (56), it becomes necessary to know the explicit formulas for the generating invariants. Also, as Example 39 shows, this final step of the group foliation method may result in a problem no easier to solve than the original differential equation.

To address these shortcomings, we replace the explicit automorphic system by a system of reconstruction equations. In essence, the reconstruction system makes use of the pseudogroup transformations to map the resolving system solution away from the cross-section, to solutions of $\Delta=0$. More precisely: a right moving frame $\rho$ will project the jet of an unknown solution along pseudogroup orbits onto the cross-section. This projection is identical to the intersection of the orbit of the solution with the cross-section, and hence is characterized as a solution of the resolving system $\mathcal{R}_{r}$ studied in the previous section. A left moving frame $\bar{\varrho}$ inverts this process, mapping a resolving system solution away from the crosssection and back to solutions of $\Delta=0$. See Figure 3 for the geometry of this process. We begin by introducing the pseudogroup jet differentials of $\mathcal{G}$, which will allow the determination of reconstruction equations in a purely symbolic manner.

4.2.1. Pseudo-group jet differentials. In this section, we introduce the pseudogroup jet differential expressions arising simply from taking the exterior derivative of the pseudogroup jets. Pull-back of these pseudogroup jet differentials by the right moving frame results in an expression for the exterior derivatives 
of the left moving frame components in terms of 'known quantities': invariant horizontal differential forms and the right moving frame pull-backs of the right Maurer-Cartan forms, computed using the universal recurrence relation (32). Expansion of these exterior derivatives in the invariant horizontal coframe yields differential equations for the left moving frame; after restriction to a resolving system solution, these differential equations become the reconstruction equations.

The pseudogroup jet differential expressions rely on the relation between left and right Maurer-Cartan forms. Recall from Section 2 the right and left zero-order Maurer-Cartan forms, respectively, for the full diffeomorphism pseudogroup:

$$
\mu^{a}=d Z^{a}-\sum_{b=1}^{m} Z_{z^{b}}^{a} d z^{b}, \quad \bar{\mu}^{a}=d z^{a}-\sum_{b=1}^{m} z_{Z^{b}}^{a} d Z^{b} .
$$

Higher-order right and left Maurer-Cartan forms $\mu_{A}^{a}=\mathbb{D}_{Z}^{A} \mu^{a}$ and $\bar{\mu}_{A}^{a}=\mathbb{D}_{z}^{A} \bar{\mu}^{a}$ are obtained via Lie differentiation with respect to, respectively,

$$
\mathbb{D}_{Z^{a}}=\sum_{b=1}^{m} z_{Z^{a}}^{b} \mathbb{D}_{z^{b}} \quad \text { and } \quad \mathbb{D}_{z^{a}}=\sum_{b=1}^{m} Z_{z^{a}}^{b} \mathbb{D}_{Z^{b}} .
$$

The Maurer-Cartan forms of a Lie pseudogroup $\mathcal{G} \subset \mathcal{D}$ are found by restricting the diffeomorphism pseudogroup Maurer-Cartan forms to the determining equations (2) and lifted determining equations (11) for $\mathcal{G}$, with the interchange $z \leftrightarrow Z, \mu \leftrightarrow \bar{\mu}$ for the left Maurer-Cartan forms.

Using the relation (14) between left and right zero-order Maurer-Cartan forms, we find the following relations among the diffeomorphism pseudogroup jets:

$$
d z^{a}=\sum_{b=1}^{m}\left(z_{Z^{b}}^{a} d Z^{b}-z_{Z^{b}}^{a} \mu^{b}\right) .
$$

Similar relations among the higher-order diffeomorphism pseudogroup jets $z_{A}^{a}$ are obtained by Lie differentiation of (60a) with respect to $\mathbb{D}_{Z^{a}}$. For example, we find, for the first-order pseudogroup jets,

$$
d z_{Z^{c}}^{a}=\sum_{b=1}^{m}\left(z_{Z^{b} Z^{c}}^{a} d Z^{b}-z_{Z^{b} Z^{c}}^{a} \mu^{b}-z_{Z^{b}}^{a} \mu_{Z^{c}}^{b}\right) .
$$

DEFINITION 41. Equations (60) and higher-order consequences are called pseudogroup jet differentials for the diffeomorphism pseudogroup. For a Lie pseudogroup $\mathcal{G} \subset \mathcal{D}$, the pseudogroup jet differentials are obtained by application of the determining system (2), with the interchange of $z$ and $Z$, and lifted determining system (11)-(60). 
REMARK 42. It will usually be more convenient to work with pseudogroup parameters instead of pseudogroup jets. The distinction is purely computational; we will illustrate both approaches in our running example.

EXAMPLE 43. We now compute the pseudogroup jet differentials for the Lie pseudogroup action (3). Applying to (60) the determining equations

$$
x_{Y}=0, \quad x_{U}=0, \quad y=Y, \quad u=\frac{U}{x_{X}},
$$

and the lifted determining equations (12) for the right Maurer-Cartan forms, we obtain

$$
\begin{aligned}
d x & =x_{X}\left(d X-\mu^{x}\right) \\
d y & =d Y \\
d u & =\frac{1}{x_{X}}\left(d X-\mu^{x}\right)-\frac{U x_{X X}}{x_{X}}\left(d U+U \mu^{x}\right) .
\end{aligned}
$$

Lie differentiation with respect to $\mathbb{D}_{X}$ gives the higher-order relations

$$
\begin{aligned}
d x_{X}= & x_{X X}\left(d X-\mu^{x}\right)-x_{X} \mu_{X}^{x} \\
d u_{X}= & \frac{-x_{X X}}{x_{X}^{2}}\left(d X-\mu^{x}\right)-\frac{1}{x_{X}} \mu_{X}^{x}-\frac{U\left(x_{X X X} x_{X}-x_{X X}^{2}\right)}{x_{X}^{2}}\left(d U+U \mu^{x}\right) \\
& -\frac{U^{2} x_{X X}}{x_{X}} \mu^{x},
\end{aligned}
$$

and so on. Writing these jet differentials in terms of the pseudogroup parameters $\bar{f}=x, \bar{f}_{X}=x_{X}, \bar{f}_{X X}=x_{X X}, \ldots$ instead offers some simplification:

$$
\begin{aligned}
& d \bar{f}=\bar{f}_{X}\left(d X-\mu^{x}\right), \\
& d \bar{f}_{X}=\bar{f}_{X X} d X-\bar{f}_{X X} \mu^{x}-\bar{f}_{X} \mu_{X}^{x}, \\
& d \bar{f}_{X X}=\bar{f}_{X X X} d X-\bar{f}_{X X X} \mu^{x}-2 \bar{f}_{X X} \mu_{X}^{x}-\bar{f}_{X} \mu_{X X}^{x},
\end{aligned}
$$

and so on. The pseudogroup jet differentials involving the jets $u, u_{X}, u_{X X}, \ldots$ may be disregarded, since they are expressible in terms of the jet parameters determined by (61).

4.2.2. Reconstruction equations. Locally, the right moving frame $\varrho\left(z^{(\infty)}\right)$ and left moving frame $\bar{\varrho}\left(z^{(\infty)}\right)$ are completely determined by their pseudogroup jet functions $\rho\left(z^{(\infty)}\right)$ and $\bar{\rho}\left(z^{(\infty)}\right)$, respectively. Since the considerations of this section are purely local, we will refer to $\rho$ and $\bar{\rho}$ as the right and left moving frames by an abuse of terminology. 
Because the right and left moving frames $\rho$ and $\bar{\rho}$ are related by pseudogroup inversion, the right moving frame pull-back of the 'inverse' pseudogroup jets $z_{A}^{a}$ produces the left moving frame pull-back of the 'regular' pseudogroup jets $Z_{A}^{a}$ :

$$
\bar{\rho}^{*}\left(z, Z^{(\infty)}\right)=\rho^{*}\left(Z, z^{(\infty)}\right) .
$$

Thus applying the right moving frame pull-back $\rho^{*}$ to the pseudogroup jet differentials will yield an expression for the differential

$$
d \bar{\rho}=\left(d\left(\bar{\rho}^{*} z\right), d\left(\bar{\rho}^{*} z_{Z^{b}}^{a}\right), \ldots\right)
$$

of the left moving frame:

$$
d \bar{\rho} \equiv \sum_{j=1}^{p} P_{j}\left(\bar{\rho}, H, I^{(\infty)}\right) \varpi^{j},
$$

where $H, I^{(\infty)}$ are the collections of normalized invariants $H^{i}=\iota\left(x^{i}\right)$, $I_{J}^{\alpha}=\iota\left(u_{J}^{\alpha}\right)$, respectively, and $z=(x, u)$ as usual. The invariants $H^{i}, I_{J}^{\alpha}$, make their appearance in (62) via the normalized Maurer-Cartan forms $\rho^{*} \mu_{A}^{a}$ and the normalized differentials $\rho^{*} d X^{i}, \rho^{*} d U^{\alpha}$. Note that these quantities may all be computed symbolically via the universal recurrence relation (32). Giving a general expression for the functions $P_{j}$ is possible but not necessary for our discussion. To apply the identity (62) to the problem of group foliation, we restrict consideration to a particular automorphic system $\mathcal{A}_{r}$ given by a choice of resolving system solution. First consider the case of full rank, $r=p$.

Let $\Delta=0$ be a $\mathcal{G}$-invariant differential equation, and suppose that a solution to a full rank resolving system $\mathcal{R}_{r}$ is given, determining the automorphic system $\mathcal{A}_{r}$. Let $J=\left\{J^{1}, \ldots, J^{p}\right\}$ be the distinguished independent invariants for the resolving system. Because of independence, invariant horizontal projections of the forms

$$
d J^{1}, \ldots, d J^{p}
$$

constitute an invariant horizontal coframe, which may be used in place of $\varpi^{1}, \ldots$, $\varpi^{p}$ in (62). Restricted to $\mathcal{A}_{r}$, (62) then yields an explicit system of differential equations for the left moving frame as a function of the distinguished invariants $J$ via projection onto this horizontal coframe:

$$
d \bar{\rho}=\sum_{j=1}^{p} Q_{j}(\bar{\rho}, J) d J^{j} .
$$

All invariants $H, I^{(\infty)}$ are expressed as functions of the distinguished invariants via the recurrence relations. The result is a system of first-order differential 
equations that must be satisfied by $\bar{\rho}$ :

$$
D_{J^{j}} \bar{\rho}=Q_{j}(\bar{\rho}, J) .
$$

We will refer to (63) or (64) as reconstruction equations.

THEOREM 44. The reconstruction equations (63) are automorphic relative to $\mathcal{G}$.

Proof. Let $\bar{\rho}_{1}$ and $\bar{\rho}_{2}$ be two solutions of the reconstruction equations (63). Then $S_{1}=\bar{\rho}_{1} \cdot(H, I)$ and $S_{2}=\bar{\rho}_{2} \cdot(H, I)$ are $p$-dimensional submanifolds with same projection onto $\mathcal{K}^{\infty}$. Since the normalized invariants $\left(H, I^{(\infty)}\right)=\iota\left(x, u^{(\infty)}\right)$ form a complete set of invariants and parameterize $\mathcal{K}^{\infty}$, the submanifolds $S_{1}$ and $S_{2}$ have the same signatures [40, 55]; that is, $\left.\left(H, I^{(\infty)}\right)\right|_{S_{1}}=\left.\left(H, I^{(\infty)}\right)\right|_{S_{2}}$. This implies that there exists a transformation $g \in \mathcal{G}$ such that $g \cdot S_{1}=S_{2}$. By construction of $S_{1}$ and $S_{2}$, this means that $g^{(\infty)} \cdot \bar{\rho}_{1}=\bar{\rho}_{2}$.

REMARK 45. As seen in (20), a left moving frame is uniquely determined by its target point. Since the solution to the reconstruction equations (64) is expressed in terms of the source coordinates (that is coordinates on the cross-section $\mathcal{K}^{(\infty)}$ ), the solution is not unique. By the automorphic property of the reconstruction solution, if $\bar{\rho}(J)$ is a particular solution, then the general solutions have the form $g^{(\infty)} \cdot \bar{\rho}(J)$, where $g^{(\infty)} \in \mathcal{G}^{(\infty)}$.

THEOREM 46. The parameterized graph

$$
\bar{\rho}(J) \cdot(H(J), I(J))=(x(J), u(J))
$$

is the graph of a solution to the differential equation $\Delta=0$.

Proof. Let $\left(H(J), I^{(\infty)}(J)\right)$ be a solution of the resolving system. By definition, this solution must come from the invariantization of some solution $\left(x, u^{(\infty)}(x)\right)$ to the differential equation $\Delta=0$. Let $\rho(x)$ be the right moving frame sending $\left(x, u^{(\infty)}(x)\right)$ onto $\left(H(J), I^{(\infty)}(J)\right)$. Suppose that $\bar{\rho}$ is a solution to the reconstruction equations. Since $\bar{\rho}$ and $\rho^{-1}$ are both solutions of the reconstruction equations, by the automorphic property there exists $g \in \mathcal{G}$ such that

$$
\bar{\rho}=g^{(\infty)} \cdot \rho^{-1} .
$$

Since $\rho^{-1}$ maps $\left(H(J), I^{(\infty)}(J)\right)$ onto the prolonged graph $\left(x, u^{(\infty)}(x)\right)$, and $g^{(\infty)}$ preserves the property of being a prolonged graph, $\bar{\rho}(J) \cdot\left(H(J), I^{(\infty)}(J)\right)$ can be identified with $\left(x, \widetilde{u}^{(\infty)}(x)\right)$ for some function $\widetilde{u}(x)$, which must be a solution of $\Delta=0$. 
By Theorem 46, to construct a solution of $\Delta=0$, we apply $\bar{\rho}$ to the graph of the resolving system solution:

$$
\bar{\rho}\left(J^{1}, \ldots, J^{p}\right) \cdot\left(H\left(J^{1}, \ldots, J^{p}\right), I\left(J^{1}, \ldots, J^{p}\right)\right)=\left(x^{1}, \ldots, x^{p}, u^{1}, \ldots, u^{q}\right),
$$

where the normalized invariants $H=\left(\iota\left(x^{1}\right), \ldots, \iota\left(x^{p}\right)\right), I=\left(\iota\left(u^{1}\right), \ldots, \iota\left(u^{q}\right)\right)$ are evaluated on the resolving system solution.

To simplify notation in the following examples, we will use the same notation for the pseudogroup parameters and their right moving frame pull-backs.

EXAMPLE 47. Continuing Example 39, we apply the reconstruction approach to obtain solutions to (52). We begin by deriving the reconstruction equations (63). Taking the right moving frame pull-back of the zero-order pseudogroup jet differential from (61) yields

$$
d \bar{f}=\bar{f}_{X} \varpi^{x},
$$

since, as found in Example 15,

$$
\rho^{*}(d X)=0 \quad \text { and } \quad \rho^{*}\left(\mu^{x}\right)=-\varpi^{x} .
$$

By duality with (43), we find

$$
\varpi^{x} \equiv\left(J^{2}-L\right) d H+d J, \quad \varpi^{y} \equiv d H,
$$

using the constraint syzygy $K=1$ and writing $L=L(H, J)$ for our choice of resolving system solution from (55). Expressing (66) in this new coframe, we obtain

$$
d \bar{f}=\left(J^{2}-L\right) \bar{f}_{X} d H+\bar{f}_{X} d J
$$

which gives the reconstruction equations for $\bar{f}(H, J), \bar{f}_{X}(H, J)$ :

$$
D_{H} \bar{f}=\left(J^{2}-L\right) \bar{f}_{X} \quad D_{J} \bar{f}=\bar{f}_{X} .
$$

These equations determine $\bar{f}, \bar{f}_{X}$, which are the only parameters needed for reconstruction. Using (55), the reconstruction equations may be written more explicitly as

$$
D_{H} \bar{f}=-\left(\frac{J^{2}}{2}+G(H)\right) D_{J} \bar{f}, \quad D_{J} \bar{f}=\bar{f}_{X},
$$

which may be solved by the method of characteristics. Acting on the graph of the resolving system solution in the cross-section (27) by the left moving frame 
determined by the reconstruction yields a solution to the nonlinear wave equation (52) given parametrically, in terms of the invariants $H$ and $J$ :

$$
x=\bar{f}(H, J), \quad y=H, \quad u=\frac{1}{\bar{f}_{X}(H, J)} .
$$

REMARK 48. The reconstruction result in Example 47 was derived in [48] using the machinery of symmetry reduction of exterior differential systems.

We now consider the reconstruction process for nonmaximal invariant rank, $r<p$. In this case, invariant horizontal projections of the forms $d J^{1}, \ldots, d J^{r}$ cannot be used as an invariant coframe in place of the invariant forms $\varpi^{i}$. To remedy this situation, we supplement the invariant forms $d J^{1}, \ldots, d J^{r}$ with $p-r$ forms $\varpi^{j_{1}}, \ldots, \varpi^{j_{p-r}}$ from the standard invariant horizontal coframe in order to form a full invariant horizontal coframe. Thus the reconstruction equations have the modified form

$$
d \bar{\rho} \equiv \sum_{j=1}^{r} Q_{j}\left(\bar{\rho}, J^{1}, \ldots, J^{r}\right) d J^{j}+\sum_{i=1}^{p-r} P_{j_{i}}\left(\bar{\rho}, J^{1}, \ldots, J^{r}\right) \varpi^{j_{i}} .
$$

We may then use $p-r$ of these equations to express the supplemental differential forms $\varpi^{j_{i}}$ in terms of the differentials of $p-r$ moving frame components $\bar{\rho}^{a_{i}}=\rho^{*}\left(z^{a_{i}}\right), i=1, \ldots, p-r$. Solutions to these nonmaximal rank reconstruction equations will then be parameterized by the invariant variables $J^{1}, \ldots, J^{r}$ in addition to the components $\bar{\rho}^{a_{1}}, \ldots, \bar{\rho}^{a_{p-r}}$. The addition of these $p-r$ 'free parameters' in the reconstruction transformations is expected; we are attempting to reconstruct the graph of a solution to $\Delta=0$, a $p$-dimensional manifold, from the graph of a resolving system solution, an $r$-dimensional manifold.

EXAMPLE 49. We return to Example 40 to illustrate reconstruction for nonmaximal rank. Recall that in this example we have the single distinguished invariant $H$, and the resolving system solution

$$
\begin{aligned}
& J(H)=G(H) \\
& L(H)=G^{\prime}(H)+G(H)^{2} .
\end{aligned}
$$

We supplement the form $d H$ with $\varpi^{x}$ so that $\left\{d H, \varpi^{x}\right\}$ is an invariant horizontal coframe. Applying the right moving frame pull-back to the first two pseudogroup jet differentials from (61) yields

$$
d \bar{f} \equiv \bar{f}_{X} \varpi^{x}, \quad d \bar{f}_{X} \equiv \bar{f}_{X X} \varpi^{x}-\bar{f}_{X} J d H .
$$


The first equation of (68) allows us to express the invariant horizontal form $\varpi^{x}$ in terms of the moving frame components:

$$
\varpi^{x} \equiv d \bar{f} / \bar{f}_{X},
$$

reducing the second equation of (68) to

$$
d \bar{f}_{X} \equiv \frac{\bar{f}_{X X}}{\bar{f}_{X}} d \bar{f}-\bar{f}_{X} J d H .
$$

The component $\bar{f}$ of the moving frame may be taken as an independent variable so that

$$
\bar{f}_{X}=\bar{f}_{X}(\bar{f}, H), \quad \bar{f}_{X X}=\bar{f}_{X X}(\bar{f}, H),
$$

and hence (69) yields differential equations for $\bar{f}_{X}$ :

$$
D_{\bar{f}} \bar{f}_{X}=\frac{\bar{f}_{X X}}{\bar{f}_{X}}, \quad D_{H} \bar{f}_{X}=-\bar{f}_{X} J .
$$

The first equation gives $\bar{f}_{X X}$ in terms of $\bar{f}_{X}$; solving the second, we find

$$
\bar{f}_{X}(\bar{f}, H)=A(\bar{f}) e^{-\int G(H) d H}=\frac{A(\bar{f})}{B(H)},
$$

where $A(\bar{f}) \neq 0, B(H)>0$ are arbitrary functions. Hence we find solutions to (57), parameterized by $\bar{f}, H$ :

$$
(x, y, u)=\left(\bar{f}, H, \frac{1}{\bar{f}_{X}}\right)=\left(\bar{f}, H, \frac{B(H)}{A(\bar{f})}\right) .
$$

In agreement with our explicit computation of the left moving frame in (30), we have $\bar{f}=x$, and conclude that $u(x, y)=B(y) / A(x)$ solves (57).

REMARK 50. Note that, due to the automorphic property of the reconstruction equations, solutions are not unique. Acting by a transformation of $\mathcal{G}$ will produce a new reconstruction solution, and hence a new solution to $\Delta=0$. This freedom of choice in the reconstruction solution can be seen in all of our examples.

EXAMPLE 51. With explicit knowledge of the left moving frame, we can see directly the equivalence of the automorphic system and reconstruction equations. In this example, we compare directly the automorphic system (56) and reconstruction equations (67) for our running example. Taking the exterior derivative of the invariants

$$
H=y, \quad J=\frac{u_{y}}{u},
$$


we obtain

$$
d H \equiv d y, \quad d J \equiv\left(\frac{u u_{x y}-u_{x} u_{y}}{u^{2}}\right) d x+\left(\frac{u u_{y y}-u_{y}^{2}}{u^{2}}\right) d y,
$$

so that, by duality,

$$
D_{H}=D_{y}-\left(\frac{u u_{y y}-u_{y}^{2}}{u u_{x y}-u_{x} u_{y}}\right) D_{x}, \quad D_{J}=\frac{u^{2}}{u u_{x y}-u_{x} u_{y}} D_{x} .
$$

Substituting the values of the left moving frame, $\bar{f}=x$ and $\bar{f}_{X}=1 / u$, and writing out the reconstruction equations (67) explicitly,

$$
D_{H} \bar{f}=\left(\frac{J^{2}}{2}-G(H)\right) D_{J} \bar{f}, \quad D_{J} \bar{f}=\bar{f}_{X},
$$

we recover the automorphic system (46).

\section{Further examples}

In this section, we apply the group foliation method to three other examples. Example 52 gives another illustration of the method for an infinite-dimensional symmetry group. Examples 54 and 55 show how the group foliation method subsumes classical symmetry reduction techniques for finding invariant and partially invariant solutions to differential equations. The symmetry groups appearing in all examples may be obtained via Lie's standard algorithm [35].

EXAMPLE 52. In this example, we solve the nonlinear Calogero wave equation [5]

$$
u_{x t}+u u_{x x}=F\left(u_{x}\right)
$$

using the group foliation method. The differential equation (70) admits the infinite-dimensional symmetry group

$$
X=x+a(t), \quad T=t, \quad U=u+a^{\prime}(t),
$$

where $a(t)$ is an arbitrary differentiable function of $t$. The Lie pseudogroup action (71) is generated by the vector fields

$$
\mathbf{v}=a(t) \frac{\partial}{\partial x}+a^{\prime}(t) \frac{\partial}{\partial u}
$$


whose prolongation is

$$
\begin{aligned}
\mathbf{v}^{(\infty)}= & a(t) \frac{\partial}{\partial x}+a_{t} \frac{\partial}{\partial u}+\left(a_{t t}-u_{x} a_{t}\right) \frac{\partial}{\partial u_{t}}-u_{x x} a_{t} \frac{\partial}{\partial u_{x t}} \\
& +\left(a_{t t t}-u_{x} a_{t t}-2 u_{x t} a_{t}\right) \frac{\partial}{\partial u_{t t}}+\cdots
\end{aligned}
$$

The recurrence relations (31) for the lifted invariants are

$$
\begin{aligned}
& d X=\Omega^{x}+\mu, \quad d T=\Omega^{t}, \\
& d U \equiv U_{X} \Omega^{x}+U_{T} \Omega^{t}+\mu_{T}, \\
& d U_{X} \equiv U_{X X} \Omega^{x}+U_{X T} \Omega^{t}, \\
& d U_{T} \equiv U_{X T} \Omega^{x}+U_{T T} \Omega^{t}+\mu_{T T}-U_{X} \mu_{T}, \\
& d U_{X X} \equiv U_{X X X} \Omega^{x}+U_{X X T} \Omega^{t}, \\
& d U_{X T} \equiv U_{X X T} \Omega^{x}+U_{X T T} \Omega^{t}-U_{X X} \mu_{T}, \\
& d U_{T T} \equiv U_{X T T} \Omega^{x}+U_{T T T} \Omega^{t}+\mu_{T T T}-U_{X} \mu_{T T}-2 U_{X T} \mu_{T}, \quad \ldots
\end{aligned}
$$

A cross-section to the pseudogroup orbits is given by

$$
X=U_{T^{k}}=0, \quad k \geqslant 0,
$$

which leads to the normalized Maurer-Cartan forms

$$
\mu=-\varpi^{x}, \quad \mu_{T}=-I_{10} \varpi^{x}, \quad \mu_{T T}=-\left(I_{11}+I_{10}^{2}\right) \varpi^{x}, \quad \ldots
$$

Substituting (73) into (72), we obtain, up to order two, the recurrence relations

$$
\begin{array}{ll}
\mathcal{D}_{x} I_{10}=I_{20}, & \mathcal{D}_{t} I_{10}=I_{11}, \\
\mathcal{D}_{x} I_{20}=I_{30}, & \mathcal{D}_{t} I_{20}=I_{21}, \\
\mathcal{D}_{x} I_{11}=I_{21}+I_{10} I_{20}, & \mathcal{D}_{t} I_{11}=I_{12}
\end{array}
$$

Eliminating $I_{21}$ from (74), we find the syzygy

$$
\mathcal{S}: \mathcal{D}_{x} I_{11}=\mathcal{D}_{t} I_{20}+I_{10} I_{20}
$$

A generating set for the algebra of differential invariants is given by

$$
t, \quad s=I_{10}, \quad K=I_{11}, \quad L=I_{20} .
$$

For $t$ and $s$ to be independent invariant variables, we require that $L \neq 0$ as

$$
d s \wedge d t \equiv L \varpi^{x} \wedge \varpi^{y} .
$$


Then, the rank-two automorphic system is

$$
\mathcal{A}_{2}: K=K(s, t), \quad L=L(s, t) .
$$

By the chain rule,

$$
\begin{aligned}
& \mathcal{D}_{x}=\left(\mathcal{D}_{x} t\right) D_{t}+\left(\mathcal{D}_{x} s\right) D_{s}=L D_{s}, \\
& \mathcal{D}_{t}=\left(\mathcal{D}_{t} t\right) D_{t}+\left(\mathcal{D}_{t} s\right) D_{s}=D_{t}+K D_{s},
\end{aligned}
$$

and, in the variables $s, t$, the syzygy (75) is equivalent to

$$
L\left(K_{s}-s\right)=L_{t}+K L_{s} .
$$

The invariantization of the differential equation (70) gives the constraint syzygy

$$
K=F(s) .
$$

Equations (76) comprise the resolving system. Substituting (76b) into (76a), we obtain the first-order partial differential equation

$$
L_{t}+F L_{s}=L\left(F_{s}-s\right)
$$

for the invariant $L$. Assuming $F(s) \neq 0$, the solution to (77) is

$$
L(s, t)=F(s) h\left(t-\int \frac{d s}{F(s)}\right) \exp \left[-\int \frac{s}{F(s)} d s\right],
$$

where $h$ is an arbitrary differentiable function. To obtain the solution to the original differential equation (70), we solve the reconstruction equation

$$
d b=\varpi^{x}+b_{T} d t=\frac{1}{L} d s+\left(b_{T}-\frac{K}{L}\right) d t,
$$

which implies that

$$
D_{s} b=\frac{1}{L} \quad \text { and } \quad b_{T}=D_{t} b+\frac{K}{L} .
$$

Hence,

$$
b(s, t)=\int \frac{d s}{L}+a(t) \quad \text { and } \quad b_{T}=-\int \frac{L_{t}}{L^{2}} d s+\frac{F(s)}{L}+a^{\prime}(t),
$$

with $L$ given in (78). Then, the solutions to (70) of invariant rank two are

$$
(x, t, u)=\bar{\rho} \cdot(0, t, 0)=\left(\int \frac{d s}{L}+a(t), t,-\int \frac{L_{t}}{L^{2}} d s+\frac{F(s)}{L}+a^{\prime}(t)\right),
$$

where $L(s, t)$ is given by (78). 
We now assume that $L=0$, and search for solutions of invariant rank one. First, the automorphic system is now given by

$$
\mathcal{A}_{1}: s=s(t), \quad K=K(t), \quad L=L(t)=0,
$$

and, by the chain rule,

$$
\mathcal{D}_{x}=0, \quad \mathcal{D}_{t}=D_{t} .
$$

Then, the recurrence relations (74) yield the syzygy

$$
D_{t} s=K
$$

while the constraint syzygy (76b) still holds. Thus, the function $s(t)$ is a solution of the ordinary differential equation

$$
D_{t} s=F(s) .
$$

From the pseudogroup jet differentials

$$
\begin{gathered}
d b=-\mu+b_{T} d t=\varpi^{x}+b_{T} d t \\
d b_{X}=-\mu_{T}+b_{T T} d t=s \varpi^{x}+b_{T T} d t,
\end{gathered}
$$

we conclude that

$$
\varpi^{x}=d b-b_{T} d t,
$$

and so the pseudogroup jets $b_{T}, b_{T T}, \ldots$ are assumed to be functions of the pseudogroup variable $b$ and the invariant $t$. From the second equation in (81), we deduce that

$$
D_{b}\left(b_{T}\right)=s, \quad b_{T T}=D_{t}\left(b_{T}\right)+s b_{T} .
$$

Hence,

$$
b_{T}(b, t)=b \cdot s(t)+f(t),
$$

where $s(t)$ is a solution of (80) and $f(t)$ is an arbitrary differentiable function. Finally, the solutions of invariant rank one are

$$
(x, t, u)=\bar{\rho} \cdot(0, t, 0)=(b, t, b \cdot s(t)+f(t)) .
$$

REMARK 53. The solution (79) also appears in [25]. It can be seen by comparison with this author's computations that the moving frame approach yields the solution in a completely systematic manner and does not require explicit formulas for the invariants $s, K$, and $L$. 
We illustrate in the next two examples the group foliation method for finitedimensional Lie groups. In Example 54, we show how the group foliation method subsumes existing algorithms for obtaining invariant [35] and partially invariant [47] solutions. In the context of finite-dimensional Lie groups, the dimension of the automorphic system is bounded between $p$ and $p+r$, where $r$ is the dimension of the Lie group. Let $p+\delta$ be the dimension of the automorphic system, $0 \leqslant \delta \leqslant r$. The number $\delta$ is called the defect of the solution generating the automorphic system. Invariant solutions have defect $\delta=0$, while partially invariant solutions satisfy $0<\delta<r$. By limiting our search to resolving systems of rank $p+\delta-r$, we discover invariant and partially invariant solutions of rank $\delta$.

Finally, Example 55 illustrates the use of the group foliation method to reduce the order of an ordinary differential equation. Foliating a second-order ordinary differential equation with respect to a two-dimensional Lie group, we obtain a resolving system of order zero, that is an algebraic equation.

EXAMPLE 54. Consider a system of equations for a transonic gas flow [47]:

$$
u_{y}-v_{x}=0, \quad u u_{x}+v_{y}=0 .
$$

To obtain an invariant solution of (82) we foliate the equations with respect to the group of dilations

$$
X=\lambda x, \quad Y=\lambda y, \quad U=u, \quad V=v,
$$

and search for invariant rank-one solutions of the resolving system. Choosing the cross-section

$$
\mathcal{K}=\{y=1\},
$$

a complete set of invariants is given by

$$
H=\iota(x), \quad I_{i, j}=\iota\left(u_{x^{i} y^{j}}\right), \quad J_{i, j}=\iota\left(v_{x^{i} y^{j}}\right) .
$$

The recurrence relations (33) yield

$$
d H=\varpi^{i}-H \varpi^{y},
$$

and

$$
\begin{array}{ll}
I_{i+1, j}=\mathcal{D}_{x} I_{i, j}, & I_{i, j+1}=\mathcal{D}_{y} I_{i, j}-(i+j) I_{i, j}, \\
J_{i+1, j}=\mathcal{D}_{x} J_{i, j}, & J_{i, j+1}=\mathcal{D}_{y} J_{i, j}-(i+j) J_{i, j} .
\end{array}
$$

Thus, a generating set of the algebra of differential invariants is given by

$$
H, \quad I, \quad J .
$$


Modulo the commutator syzygies induced by the commutator relation

$$
\left[\mathcal{D}_{y}, \mathcal{D}_{x}\right]=\mathcal{D}_{x},
$$

there is no fundamental syzygy.

Searching for an order-one invariant solution, the automorphic system is

$$
\mathcal{A}_{1}: \quad I=I(H), \quad J=J(H) .
$$

By the chain rule,

$$
\mathcal{D}_{x}=\left(\mathcal{D}_{x} H\right) D_{H}=D_{H}, \quad \mathcal{D}_{y}=\left(\mathcal{D}_{y} H\right) D_{H}=-H D_{H} .
$$

Thus, the invariantization of the differential equations (82) yields

$$
H D_{H} I+D_{H} J=0, \quad I D_{H} I-H D_{H} J=0 .
$$

Omitting the constant solution, the integration of the resolving system gives

$$
I(H)=-H^{2}, \quad J(H)=\frac{2}{3} H^{3}+C,
$$

where $C$ is an arbitrary constant. Implementing the reconstruction step, we obtain the reconstruction equation

$$
\varpi^{y}=\frac{d \bar{\lambda}}{\bar{\lambda}}
$$

Viewing $\bar{\lambda}$ as an independent variable, the invariant solution is given by

$$
(x, y, u, v)=\bar{\lambda} \cdot(H, 1, I, J)=\left(H \bar{\lambda}, \bar{\lambda},-H^{2}, \frac{2}{3} H^{3}+C\right) .
$$

Since $\bar{\lambda}=y, H=x / \bar{\lambda}=x / y$, and the solution invariant under the dilation group (83) is

$$
u(x, y)=-\left(\frac{x}{y}\right)^{2}, \quad v(x, y)=\frac{2}{3}\left(\frac{x}{y}\right)^{3}+C .
$$

We now obtain a partially invariant solution of (54) by foliating (82) with respect to

$$
X=\lambda x, \quad Y=\lambda y, \quad U=u, \quad V=v+\epsilon .
$$

This time, a cross-section is given by

$$
\mathcal{K}=\{y=1, v=0\},
$$

and

$$
H=\iota(x), \quad I=\iota(u), \quad J=\iota\left(v_{x}\right), \quad K=\iota\left(v_{y}\right),
$$


form a generating set of invariants. These invariants admit one fundamental syzygy

$$
\mathcal{D}_{y} J=\mathcal{D}_{x} K+J .
$$

Restricting ourself to the rank-one automorphic system

$$
I=I(H), \quad J=J(H), \quad K=K(H),
$$

the corresponding resolving system is

$$
J+H D_{H} I=0, \quad K+I D_{H} I=0, \quad D_{H}\left[\left(H^{2}+I\right) D_{H} I\right]=0,
$$

where the first two equations come from the invariantization of (82) and the third equation is a consequence of syzygy (87). Hence, provided $I(H)$ is a solution of

$$
\left(H^{2}+I\right) D_{H} I=C,
$$

where $C$ is a constant, the invariants $J$ and $K$ are completely determined by (88). Implementing the reconstruction step, the first of two reconstruction equations is given by (85). From (84), which still holds, we conclude that

$$
\varpi^{x}=d H+H \frac{d \bar{\lambda}}{\bar{\lambda}} .
$$

Hence, integrating the second reconstruction equation

$$
d \bar{\epsilon}=J \varpi^{x}+K \varpi^{y}=-H D_{H} I d H-C \frac{d \bar{\lambda}}{\bar{\lambda}},
$$

we obtain

$$
\bar{\epsilon}=-C \ln \bar{\lambda}-\int H D_{H} I d H .
$$

This produces the partially invariant solution

$$
\begin{aligned}
(x, y, u, v) & =(\bar{\lambda}, \bar{\epsilon}) \cdot(H, 1, I, 0) \\
& =\left(H \bar{\lambda}, \bar{\lambda}, I(H),-C \ln \bar{\lambda}-\int H D_{H} I d H\right) .
\end{aligned}
$$

Since $H=x / y$ and $\bar{\lambda}=y$,

$$
u(x, y)=I(x / y), \quad v(x, y)=-C \ln y-\int(x / y) I^{\prime}(x / y) d(x / y) .
$$


EXAMPLE 55. Consider the nonlinear second-order ordinary differential equation

$$
x^{2} u_{x x}=\left(x u_{x}-u\right)^{2}, \quad x>0 .
$$

The equation (89) is invariant under the two-dimensional solvable group of transformations

$$
X=\lambda x, \quad U=u+\epsilon x \quad \text { with } \lambda>0 \text { and } \epsilon \in \mathbb{R} .
$$

In the pseudogroup framework, the determining equations of the Lie group action (90) are

$$
x X_{x}=X, \quad X_{u}=0, \quad x U_{x}=U-u, \quad U_{u}=1,
$$

and the infinitesimal determining equations corresponding to an infinitesimal generator $\mathbf{v}=\xi(x, u) \partial_{x}+\phi(x, u) \partial_{u}$ are

$$
x \xi_{x}=\xi, \quad \xi_{u}=0, \quad x \phi_{x}=\phi, \quad \phi_{u}=0 .
$$

Hence, the general prolonged infinitesimal generator is

$$
\mathbf{v}=\xi_{x}\left(x \frac{\partial}{\partial x}-\sum_{k=1}^{\infty} k u_{x^{k}} \frac{\partial}{\partial u_{x^{k}}}\right)+\phi_{x}\left(x \frac{\partial}{\partial u}+\frac{\partial}{\partial u_{x}}\right),
$$

and the order-zero lifted recurrence relations are

$$
d X=\Omega^{x}+X \mu_{X}^{x}, \quad d U \equiv U_{X} \Omega^{x}+X \mu_{X}^{u} .
$$

Choosing the cross-section $\mathcal{K}^{0}=\{x=1, u=0\}$, the recurrence relations (92) yield the normalized Maurer-Cartan forms

$$
\mu_{X}^{x}=-\varpi^{x}, \quad \mu_{X}^{u} \equiv-I_{1} \varpi^{x} .
$$

Now, let

$$
\mathcal{A}_{1}: z=I_{1}=\iota\left(u_{x}\right)=x u_{x}-u, \quad v(z)=I_{2}=\iota\left(u_{x x}\right)=x^{2} u_{x x}
$$

be the rank-one automorphic system, which requires that

$$
v \neq 0
$$

as $d z \equiv v \varpi^{x}$. Since there are no syzygies, the invariantization of the differential equation (89) yields the resolving system

$$
v(z)=z^{2} .
$$


Hence, the constraint (93) is satisfied, provided that $z \neq 0$. When this is so, $\omega^{x}=$ $d z / v$, and the reconstruction equations are

$$
D_{z}(x)=\frac{x}{z^{2}}, \quad D_{z}(u)=\frac{u}{z^{2}}+\frac{1}{z} .
$$

Solving (94), we obtain

$$
x(z)=A e^{-1 / z}, \quad u(z)=e^{-1 / z}\left[\int \frac{e^{1 / z}}{z} d z+B\right],
$$

where $A$ and $B$ are two constants. By construction, the parametric curve (95) is a solution of (89); to recover the solution in the form $u(x)$ it suffices to express the parameter $z$ as a function of $x$ using the first equation in (95):

$$
u(x)=-x\left[\int \frac{d x}{x^{2}(\ln x+A)}+B\right] .
$$

REMARK 56. When $\mathcal{G}$ is a (local) Lie group action as in Example 55, we can rely on the abstract definition of Lie groups to obtain a simple expression for the reconstruction equations. By Ado's theorem [18], every Lie group is locally isomorphic to some linear group $\mathcal{G} \simeq G \subset \mathrm{GL}(k)$ for some $k \in \mathbb{N}$, and a right moving frame is a $G$-equivariant map $\rho: \mathrm{J}^{n} \rightarrow G$ satisfying

$$
\rho\left(g \cdot z^{(n)}\right)=\rho\left(z^{(n)}\right) \cdot g^{-1} .
$$

As for Lie pseudogroups, the corresponding left moving frame is obtained by group inversion $\bar{\rho}=\rho^{-1}$, and the reconstruction equations (62) are equivalent to

$$
d \bar{\rho}=d \rho^{-1}=-\bar{\rho} \cdot\left(d \rho \cdot \rho^{-1}\right)=-\bar{\rho} \mu,
$$

where $\mu$ is the moving frame pulled-back Lie algebra valued right-invariant Maurer-Cartan form of $G$ (restricted to a solution of the resolving system). Examples of integrating ordinary differential equations using this point of view can be found in [28, Ch. 6].

\section{Normal subpseudogroups}

For pseudogroups admitting normal subpseudogroups, it is possible to split the reconstruction procedure into a series of subreconstruction steps involving smaller pseudogroups. This is the moving frame version of Vessiot's observation [56] that the integration of an automorphic system can be replaced by the integration of a sequence of differential equations automorphic with respect to primitive simple Lie pseudogroups.

Definition 57. A subpseudogroup $\mathcal{H} \subseteq \mathcal{G}$ is normal if, for all $h \in \mathcal{H}$ and $g \in \mathcal{G}$, 


$$
g \circ h \circ g^{-1} \in \mathcal{H}
$$

whenever the composition is defined.

Let $\mathfrak{h}$ and $\mathfrak{g}$ denote the Lie algebras of $\mathcal{H}$ and $\mathcal{G}$, respectively. Infinitesimally, if $\mathcal{H}$ is a normal subpseudogroup of $\mathcal{G}$, then $\mathfrak{h}$ is an ideal of $\mathfrak{g}$ :

$$
[\mathfrak{h}, \mathfrak{g}] \subseteq \mathfrak{h} .
$$

EXAMPLE 58. To illustrate Definition 57, we introduce the Lie pseudogroups

$$
\mathcal{H}: X=x, \quad Y=y+g(x), \quad U=u+g^{\prime}(x),
$$

and

$$
\mathcal{G}: X=f(x), \quad Y=f^{\prime}(x) y+g(x), \quad U=u+\frac{f^{\prime \prime}(x) y+g^{\prime}(x)}{f^{\prime}(x)},
$$

where $f(x) \in \mathcal{D}(\mathbb{R})$ is a local diffeomorphism and $g(x)$ is an arbitrary smooth function. The pseudogroup $\mathcal{H}$ is a subpseudogroup of $\mathcal{G}$ obtained by setting $f=\mathbb{1}$ to be the identity map in (98). To verify (97), let

$$
g \cdot(x, y, u)=\left(f(x), f^{\prime}(x) y+g(x), u+\frac{f^{\prime \prime}(x) y+g^{\prime}(x)}{f^{\prime}(x)}\right) \in \mathcal{G}
$$

and

$$
g^{-1} \cdot(X, Y, U)=\left(F(X), F^{\prime}(X) Y+G(X), U+\frac{F^{\prime \prime}(X) Y+G^{\prime}(X)}{F^{\prime}(X)}\right),
$$

where $F(X)=f^{-1}(X)$ and $G(X)=-g(F(X)) / f^{\prime}(F(X))$. If

$$
h \cdot(x, y, u)=\left(x, y+h(x), u+h^{\prime}(x)\right) \in \mathcal{H},
$$

a direct computation shows that

$$
g \circ h \circ g^{-1} \cdot(X, Y, U)=\left(X, Y+H(X), U+H^{\prime}(X)\right) \in \mathcal{H},
$$

with $H(X)=f^{\prime}(F(X)) \cdot h(F(X))$. Infinitesimally, the Lie algebras of $\mathcal{G}$ and $\mathcal{H}$ are spanned by

$$
\begin{aligned}
& \mathfrak{g}=\operatorname{span}\left\{\mathbf{v}_{a}=a(x) \frac{\partial}{\partial x}+y a^{\prime}(x) \frac{\partial}{\partial y}+y a^{\prime \prime}(x) \frac{\partial}{\partial u}, \mathbf{w}_{b}=b(x) \frac{\partial}{\partial y}+b^{\prime}(x) \frac{\partial}{\partial u}\right\}, \\
& \mathfrak{h}=\operatorname{span}\left\{\mathbf{w}_{b}=b(x) \frac{\partial}{\partial y}+b^{\prime}(x) \frac{\partial}{\partial u}\right\}
\end{aligned}
$$


where $a(x)$ and $b(x)$ are arbitrary smooth functions. Computing the basic commutators

$$
\left[\mathbf{v}_{a}, \mathbf{v}_{b}\right]=\mathbf{v}_{a b^{\prime}-b a^{\prime}}, \quad\left[\mathbf{w}_{a}, \mathbf{w}_{b}\right]=0, \quad\left[\mathbf{v}_{a}, \mathbf{w}_{b}\right]=\mathbf{w}_{a b^{\prime}-b a^{\prime}},
$$

we see that $\mathfrak{h}$ is an abelian ideal of $\mathfrak{g}$.

Given a normal Lie subpseudogroup $\mathcal{H}$ of $\mathcal{G}$, the definition of the quotient Lie pseudogroup of $\mathcal{G}$ by $\mathcal{H}$ is based on the notion of invariant admissible fibration introduced by Rodrigues in [49]. We now recast the main definitions of [49] at the pseudogroup level. Given a fibered manifold $\pi: M \rightarrow N$ and a Lie pseudogroup $\mathcal{G}$ acting on $M$, a local diffeomorphism $g \in \mathcal{G}$ is said to be projectable by $\pi$ if there exists a local diffeomorphism $\varphi \in \mathcal{D}(N)$ such that $\pi \circ g=\varphi \circ \pi$. We denote by $\tilde{\pi}(g)=\varphi$ the map that sends the projectable diffeomorphism $g$ to its projection $\varphi$. The fibration $\pi: M \rightarrow N$ is said to be $\mathcal{G}$-invariant if every pseudogroup transformation $g \in \mathcal{G}$ is projectable.

Recall from Definition 1 that the map $\pi_{n}^{n+k}: \mathcal{G}^{(n+k)} \rightarrow \mathcal{G}^{(n)}$ denotes the standard pseudogroup jet projection.

Definition 59. A $\mathcal{G}$-invariant fibration $\pi: M \rightarrow N$ is called $\mathcal{G}$-admissible if there are integers $n_{0}$ and $k_{0}$ such that $(\operatorname{ker} \tilde{\pi})^{(n)} \cap \mathcal{G}^{(n)}$ and

$$
\pi_{n}^{n+k}\left((\operatorname{ker} \tilde{\pi})^{(n+k)} \cap \mathcal{G}^{(n+k)}\right)
$$

are subbundles of the pseudogroup jet bundle $\mathcal{G}^{(n)}$ for $n \geqslant n_{0}$ and $k \geqslant k_{0}$.

Definition 60. Let $\mathcal{G}$ and $\mathcal{H}$ be two Lie pseudogroups acting on $M$ and $N$, respectively. A homomorphism of $\mathcal{G}$ onto $\mathcal{H}$ is a fibration $\pi: M \rightarrow N$ which is $\mathcal{G}$-admissible and such that $\tilde{\pi}(\mathcal{G})=\mathcal{H}$. If the kernel ker $\tilde{\pi}$ is trivial, then $\tilde{\pi}$ is said to be an isomorphism of $\mathcal{G}$ onto $\mathcal{H}$.

Definition 61. Let $\mathcal{H}$ be a normal sub-Lie pseudogroup of $\mathcal{G}$. A Lie pseudogroup $\mathcal{Q}$ is a quotient of $\mathcal{G}$ by $\mathcal{H}$ if there exist Lie pseudogroups $\widetilde{\mathcal{G}}$ and $\widetilde{\mathcal{H}} \subset \widetilde{\mathcal{G}}$, an isomorphism $\widetilde{\pi}: \widetilde{\mathcal{G}} \rightarrow \mathcal{G}$ such that $\widetilde{\pi}(\widetilde{\mathcal{H}})=\mathcal{H}$, and a homomorphism $\widetilde{\beta}: \widetilde{\mathcal{G}} \rightarrow \mathcal{Q}$ whose kernel is $\widetilde{\mathcal{H}}$.

Pictorially, we have the following.




The last three definitions naturally fit within the moving frame framework. Given a Lie pseudogroup $\mathcal{G}$ acting on $M$ and a normal Lie subpseudogroup $\mathcal{H} \subset \mathcal{G}$, consider their isomorphic prolongations $\mathcal{G}^{(\infty)}$ and $\mathcal{H}^{(\infty)}$ obtained by considering their prolonged action on the set of regular jets $\mathcal{V}^{\infty} \subset \mathrm{J}^{\infty}$ of $\mathcal{G}^{(\infty)}$. Let $\mathcal{K}_{\mathcal{H}}$ be a cross-section to the $\mathcal{H}^{(\infty)}$-orbits, and let $\varrho_{\mathcal{H}}$ be the corresponding right moving frame. Then, the projection $\tau \circ \varrho_{\mathcal{H}}: \mathcal{V}^{\infty} \rightarrow \mathcal{K}_{\mathcal{H}}$ onto the crosssection $\mathcal{K}_{\mathcal{H}}$ is a $\mathcal{G}^{(\infty)}$-invariant admissible fibration of $\mathcal{V}^{\infty}$. The quotient of $\mathcal{G}$ by $\mathcal{H}$ can then be identified with the projected action of $\mathcal{G}^{(\infty)}$ onto $\mathcal{K}_{\mathcal{H}}$ which we will write as $\mathcal{G}^{(\infty)} / \mathcal{H}^{(\infty)}$.

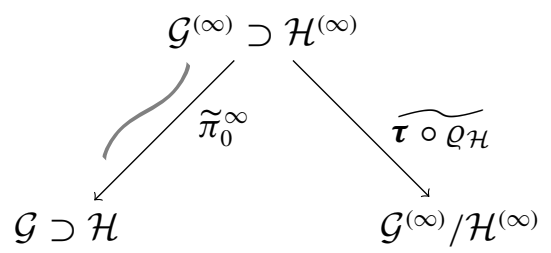

Since $\mathcal{K}_{\mathcal{H}}$ can be identified with the space of $\mathcal{H}$-invariants, the quotient pseudogroup $\mathcal{G}^{(\infty)} / \mathcal{H}^{(\infty)}$ has a well-defined action on the space of $\mathcal{H}$-invariants. Finally, we note that the quotient pseudogroup $\mathcal{G}^{(\infty)} / \mathcal{H}^{(\infty)}$ is isomorphic [51] to the subpseudogroup of transformations of $\mathcal{G}$ that keep the cross-section $\mathcal{K}_{\mathcal{H}}$ invariant:

$$
\mathcal{G} / \mathcal{H}=\left\{g \in \mathcal{G} \mid g^{(\infty)} \cdot \mathcal{K}_{\mathcal{H}} \in \mathcal{K}_{\mathcal{H}}\right\} \subset \mathcal{G} .
$$

EXAMPLE 62. Continuing Example 58, we now implement the moving frame method for the normal subpseudogroup $\mathcal{H}$. Up to order two, the lifted invariants are

$$
\begin{gathered}
X=x, \quad Y=y+g(x), \quad U=u+g_{x}, \\
U_{X}=u_{x}+g_{x x}-g_{x} u_{y}, \quad U_{Y}=u_{y}, \\
U_{X X}=u_{x x}+g_{x x x}-g_{x x} u_{y}-2 g_{x} u_{x y}+g_{x}^{2} u_{y y}, \\
U_{X Y}=u_{x y}-g_{x} u_{y y}, \quad U_{Y Y}=u_{y y} .
\end{gathered}
$$

Choosing the cross-section

$$
\mathcal{K}_{\mathcal{H}}^{\infty}=\left\{y=u_{x^{k}}=0, k \geqslant 0\right\},
$$

we find, up to order three, the invariants

$$
\begin{gathered}
X=x, \quad I_{01}=u_{y}, \quad I_{11}=u_{x y}+u u_{y y}, \quad I_{02}=u_{y y}, \\
I_{21}=u_{x x y}+\left(u_{x}+u u_{y}\right) u_{y y}+2 u u_{x y y}+u^{2} u_{y y y}, \\
I_{12}=u_{x y y}+u u_{y y y}, \quad I_{03}=u_{y y y} .
\end{gathered}
$$


We now introduce the quotient pseudogroup $\mathcal{G}^{(\infty)} / \mathcal{H}^{(\infty)}$ acting on the $\mathcal{H}$-invariants (101):

$$
\begin{gathered}
X=f(x), \quad J_{01}=\frac{I_{01}}{f_{x}}+\frac{f_{x x}}{f_{x}^{2}}, \quad J_{11}=\frac{I_{11}}{f_{x}^{2}}+\frac{f_{x x x}-f_{x x} I_{01}}{f_{x}^{3}}-2 \frac{f_{x x}^{2}}{f_{x}^{4}}, \\
J_{02}=\frac{I_{02}}{f_{x}^{2}}, \quad J_{12}=\frac{f_{x} I_{12}-2 f_{x x} I_{02}}{f_{x}^{4}}, \quad J_{03}=\frac{I_{03}}{f_{x}^{3}}, \quad \ldots
\end{gathered}
$$

In the above action formulas, $J_{i, j}$ denotes the image of the invariant $I_{i, j}$. This pseudogroup is the prolongation to the $\mathcal{H}$-invariants of the subpseudogroup

$$
\mathcal{G} / \mathcal{H}: X=f(x), \quad Y=f^{\prime}(x) y, \quad U=u+\frac{f^{\prime \prime}(x) y}{f^{\prime}(x)}
$$

of $\mathcal{G}$ that fixes the cross-section (100). The pseudogroup (103) originally appeared in [31], where Medolaghi systematically studies isomorphic representations of the diffeomorphism pseudogroup $\mathcal{D}(\mathbb{R})$. This pseudogroup was also used by Vessiot [56] in his work on automorphic systems.

Given a differential equation $\Delta=0$ with symmetry pseudogroup $\mathcal{G}$, if we assume that $\mathcal{H} \subset \mathcal{G}$ is a normal subpseudogroup, then it is possible to apply the group foliation procedure iteratively. First, we foliate the solution space of the differential equation with respect to the normal subpseudogroup $\mathcal{H}$, and project solutions onto the cross-section $\mathcal{K}_{\mathcal{H}}$ defining a moving frame for $\mathcal{H}$. Let $\mathcal{A}_{r}^{\mathcal{H}}$ and $\mathcal{R}_{r}^{\mathcal{H}}$ be the corresponding automorphic and resolving systems.

PROPOSITION 63. The resolving system $\mathcal{R}_{r}^{\mathcal{H}}$ is invariant under the quotient pseudogroup $\mathcal{G} / \mathcal{H}$ (to be more accurate, we should write $\mathcal{G}^{(\infty)} / \mathcal{H}^{(\infty)}$ instead of $\mathcal{G} / \mathcal{H}$, but, since the two pseudogroups are isomorphic, from now on, we use the latter to simplify the notation).

Proof. Since syzygies among differential invariants are invariant under the diffeomorphism pseudogroup $\mathcal{D}(M)$, it follows that $\mathcal{R}_{r}^{\mathcal{H}}$ is invariant under the quotient pseudogroup $\mathcal{G} / \mathcal{H}$ as the differential equation $\Delta=0$ is $\mathcal{G}$-invariant.

The invariance of resolving system $\mathcal{R}_{r}^{\mathcal{H}}$ under $\mathcal{G} / \mathcal{H}$ permits us to foliate the solution space of $\mathcal{R}_{r}^{\mathcal{H}}$ with respect to the quotient pseudogroup $\mathcal{G} / \mathcal{H}$. The result is the same as foliating the differential equation $\Delta=0$ by the full symmetry pseudogroup $\mathcal{G}$.

Assuming that a solution to the resolving system $\mathcal{R}_{\widetilde{r}}^{\mathcal{G}}$ is known, Figure 4 shows that the reconstruction operation splits into two steps. First, we can solve the 




Figure 4. Iterative group foliation.

reconstruction equations for the quotient pseudogroup $\mathcal{G} / \mathcal{H}$ to obtain a solution to the resolving system $\mathcal{R}_{r}^{\mathcal{H}}$. Given a solution, solving the reconstruction equations for the normal pseudogroup $\mathcal{H}$ yields a solution to the original differential equation. At the level of moving frames, this reflects the fact that the left $\mathcal{G}$ equivariant moving frame is equivalent to the composition

$$
\bar{\rho}_{\mathcal{G}}=\bar{\rho}_{\mathcal{H}} \cdot \bar{\rho}_{\mathcal{G} / \mathcal{H}} \cdot
$$

In general, the reconstruction procedure can split into many steps. Given a Lie pseudogroup $\mathcal{G}$, let $\mathcal{G}_{1} \subsetneq \mathcal{G}$ be a proper maximal normal sub-Lie pseudogroup of $\mathcal{G}$. Similarly, let $\mathcal{G}_{2} \subsetneq \mathcal{G}_{1}$ be a proper maximal normal sub-Lie pseudogroup of $\mathcal{G}_{1}$. Repeating the procedure, assume that it is possible to obtain a finite chain of sub-Lie pseudogroups,

$$
\{\mathbb{1}\}=\mathcal{G}_{\ell+1} \subsetneq \mathcal{G}_{\ell} \subsetneq \mathcal{G}_{\ell-1} \subsetneq \cdots \subsetneq \mathcal{G}_{1} \subsetneq \mathcal{G}_{0}=\mathcal{G},
$$

such that, for each $k=0, \ldots, \ell$, the quotient $\overline{\mathcal{G}}_{k}=\mathcal{G}_{k} / \mathcal{G}_{k+1}$ is a simple pseudogroup. Then, the group foliation method reduces to solving the resolving system $\mathcal{R}_{r}^{\mathcal{G}}$ followed by a series of reconstruction steps for the simple sub-Lie pseudogroups $\overline{\mathcal{G}}_{\ell}, \ldots, \overline{\mathcal{G}}_{0}$. According to Cartan [6], each subreconstruction step will only require the integration of either ordinary differential equations or linear partial differential equations involving no more than one arbitrary function.

EXAMPLE 64. To illustrate the iterative reconstruction procedure, we foliate the differential equation

$$
u_{x y y}+u u_{y y y}+2 u_{y} u_{y y}=0
$$

with respect to the symmetry pseudogroup (98). The moving frame construction for this Lie pseudogroup can be found in $[42,43]$. Choosing the cross-section

$$
\mathcal{K}_{\mathcal{G}}^{\infty}=\left\{x=y=u_{x^{k}}=u_{y x^{k}}=0, u_{y y}=1: k \geqslant 0\right\},
$$

and letting $I_{i j}=\iota\left(u_{x^{i} y^{j}}\right)$ and $\mu_{k}=\iota\left(a_{k}\right), v_{k}=\iota\left(b_{k}\right)$, where $a(x), b(x)$ are the arbitrary functions occurring in the infinitesimal generators (99), the normalized Maurer-Cartan forms of order $\leqslant 2$ are

$\mu \equiv-\varpi^{x}, \quad \mu_{X} \equiv \frac{1}{2}\left(I_{12} \varpi^{x}+I_{03} \varpi^{y}\right), \quad \mu_{X X} \equiv v \equiv-\varpi^{y}, \quad v_{X} \equiv v_{X X} \equiv 0$. 
The recurrence relations for the third-order normalized invariants are

$$
\begin{array}{ll}
\mathcal{D}_{x} I_{12}=I_{22}-\frac{3}{2} I_{12}^{2}, & \mathcal{D}_{y} I_{12}=I_{13}-\frac{3}{2} I_{12} I_{03}+2, \\
\mathcal{D}_{x} I_{03}=I_{13}-\frac{3}{2} I_{12} I_{03}, & \mathcal{D}_{y} I_{03}=I_{04}-\frac{3}{2} I_{03}^{2},
\end{array}
$$

while the fundamental syzygy, modulo the commutator relation

$$
\left[\mathcal{D}_{x}, \mathcal{D}_{y}\right]=\frac{I_{03}}{2} \mathcal{D}_{x}-\frac{I_{12}}{2} \mathcal{D}_{y},
$$

is given by

$$
\mathcal{D}_{x} I_{03}-\mathcal{D}_{y} I_{12}=-2 .
$$

Implementing the group foliation algorithm, we let the third-order normalized invariants

$$
s=\iota\left(u_{x y y}\right), \quad t=\iota\left(u_{y y y}\right),
$$

play the role of the independent invariants, and let the fourth-order normalized invariants

$$
I_{22}(s, t)=\iota\left(u_{x^{2} y^{2}}\right), \quad I_{13}(s, t)=\iota\left(u_{x y^{3}}\right), \quad I_{04}(s, t)=\iota\left(u_{y^{4}}\right)
$$

be the dependent invariants so that (110) forms the automorphic system. Then, the invariantization of (104) yields

$$
s=0 .
$$

Hence, the automorphic system (110) will produce invariant rank-one solutions. Taking into account (111) the fundamental syzygy (109) yields

$$
\mathcal{D}_{x} t=-2 \text {. }
$$

By the chain rule,

$$
\mathcal{D}_{x}=\left(\mathcal{D}_{x} s\right) D_{s}+\left(\mathcal{D}_{x} t\right) D_{t}=-2 D_{t}, \quad \mathcal{D}_{y}=\left(\mathcal{D}_{y} s\right) D_{s}+\left(\mathcal{D}_{y} t\right) D_{t}=I D_{t},
$$

where $I(t)=\mathcal{D}_{y} t=I_{04}-3 t^{2} / 2$ by the recurrence relations (107). Differentiating the fundamental syzygy (112) with respect to $\mathcal{D}_{y}$, and using the commutation relation (108), we deduce the differential equation

$$
2 D_{t} I=t \quad \text { or } \quad D_{t} I_{04}=\frac{7}{2} t .
$$

On the other hand, the recurrence relations (107) imply that

$$
I_{22}=0 \quad \text { and } \quad I_{13}=-2 .
$$


In summary, the equations (111), (113), and (114) form the resolving system. Integrating (113), we obtain

$$
I(t)=\frac{a^{2}+t^{2}}{4} \quad \text { or } \quad I_{04}(t)=\frac{a^{2}+7 t^{2}}{4}
$$

where $a$ is a constant of integration.

We are now ready to implement the reconstruction procedure. Based on Examples 58 and 62, we first implement the reconstruction procedure for the quotient pseudogroup (102). Using the Maurer-Cartan normalizations (106), the reconstruction equations (62) for the quotient action are, up to order two,

$$
\begin{gathered}
d \bar{f}=\bar{f}_{X} \varpi^{x}, \quad d \bar{f}_{X}=\bar{f}_{X X} \varpi^{x}-\frac{t}{2} \bar{f}_{X} \varpi^{y}, \\
d \bar{f}_{X X}=\bar{f}_{X X X} \varpi^{x}+\left(\bar{f}_{X}-t \bar{f}_{X X}\right) \varpi^{y} .
\end{gathered}
$$

From the first equation, we have that

$$
\varpi^{x}=d \bar{f} / \bar{f}_{X} .
$$

On the other hand, from the equality

$$
d t=\left(\mathcal{D}_{x} t\right) \varpi^{x}+\left(\mathcal{D}_{y} t\right) \varpi^{y}=-2 \varpi^{x}+I \varpi^{y},
$$

we have that

$$
\varpi^{y}=\frac{1}{I}\left(d t+2 \frac{d \bar{f}}{\bar{f}_{X}}\right) .
$$

The second and third equations of (115) then reduce to

$$
\begin{aligned}
& D_{t} \bar{f}_{X}=-\frac{t \bar{f}_{X}}{2 I}, \quad D_{\bar{f}} \bar{f}_{X}=\frac{\bar{f}_{X X}}{\bar{f}_{X}}-\frac{t}{I}, \\
& D_{t} \bar{f}_{X X}=\frac{\bar{f}_{X}-t \bar{f}_{X X}}{I}, \quad D_{\bar{f}} \bar{f}_{X X}=\frac{\bar{f}_{X X X}}{\bar{f}_{X}}+\frac{2\left(\bar{f}_{X}-t \bar{f}_{X X}\right)}{I \bar{f}_{X}} .
\end{aligned}
$$

From (118a), we deduce that

$$
\bar{f}_{X}(t, \bar{f})=\frac{F(\bar{f})}{a^{2}+t^{2}}, \quad \bar{f}_{X X}(t, \bar{f})=\frac{F(\bar{f})\left(F^{\prime}(\bar{f})-4 t\right)}{\left(a^{2}+t^{2}\right)^{2}},
$$

where $F(\bar{f}) \neq 0$ is an arbitrary nonzero smooth function. At the next order, substituting (119) into the first equation of (118b), we see that the first equation is identically satisfied and the second equation of (118b) defines $\bar{f}_{X X X}$. Continuing 
the computation at higher order, we obtain the expressions for $\bar{f}_{X^{k}}, k \geqslant 4$, giving the left moving frame $\bar{\rho}_{\mathcal{G} / \mathcal{H}}$.

The next step in the iterative reconstruction procedure is to construct the left moving frame $\bar{\rho}_{\mathcal{H}}$. To obtain the reconstruction equations for the pseudogroup jets $\bar{g}, \bar{g}_{X}, \ldots$, we first compute (60) for the full symmetry pseudogroup (98), and then restrict the equations to the cross-section (100). This yields

$$
d \bar{g}=\bar{g}_{X} \varpi^{x}+\bar{f}_{X} \varpi^{y}, \quad d \bar{g}_{X}=\bar{g}_{X X} \varpi^{x}+\left(\bar{f}_{X X}-\frac{t \bar{g}_{X}}{2}\right) \varpi^{y}, \quad \ldots
$$

Using (116), (117), and (119), we obtain

$$
D_{t} \bar{g}=\frac{4 F(\bar{f})}{\left(t^{2}+a^{2}\right)^{2}}, \quad \frac{\bar{g}_{X}}{\bar{f}_{X}}=D_{\bar{f}} \bar{g}-\frac{2}{I} .
$$

Integrating the first equation, we find that

$$
\begin{aligned}
& \bar{g}=4 F(\bar{f})\left(\frac{t}{2 a^{2}\left(t^{2}+a^{2}\right)}+\frac{1}{2 a^{3}} \arctan \frac{t}{a}\right)+G(\bar{f}), \\
& \frac{\bar{g}_{X}}{\bar{f}_{X}}=4 F^{\prime}(\bar{f})\left(\frac{t}{2 a^{2}\left(t^{2}+a^{2}\right)}+\frac{1}{2 a^{3}} \arctan \frac{t}{a}\right)+G^{\prime}(\bar{f})-\frac{8}{t^{2}+a^{2}},
\end{aligned}
$$

where $G(\bar{f})$ is an arbitrary smooth function. Thus, a parameterized solution to the partial differential equation (104) is given by

$$
(x, y, u)=\bar{\rho}_{\mathcal{G}}=\bar{\rho}_{\mathcal{H}} \cdot \bar{\rho}_{\mathcal{G} / \mathcal{H}} \cdot(0,0,0)=\left(\bar{f}, \bar{g}, \frac{\bar{g}_{X}}{\bar{f}_{X}}\right) .
$$

\section{Conclusion}

Using the machinery of equivariant moving frames, we have attempted to provide a unified and computationally clear approach to group foliation. The newness and broad applicability of moving frame theory brings fresh insight to old algorithms, and, as such, many unexplored directions present themselves. We list here several possibilities for further research.

(a) One of the most obvious applications of group foliation is to the solution of differential equations. There are many physically interesting equations that may be particularly amenable to our version of group foliation because of the complexity of their symmetry pseudogroups. Four particularly interesting examples are the following: the Davey-Stewartson equations [7], the InfeldRowlands equation [12], the potential Kadomtsev-Petviashvili equation [11], and the Calabi-Yau equation for Kähler-Einstein metrics [57]. 
(b) The results and algorithms presented in the present paper relied on the construction of a moving frame. By appropriately adapting the exposition, it is possible to encompass the situation where only a partial moving frame $[46,55]$ exists on the solution space of a differential equation. For example, when foliating (52) with respect to its full symmetry group

$$
X=f(x), \quad Y=g(y), \quad U=\frac{u}{f^{\prime}(x) g^{\prime}(y)},
$$

no differential invariants exist on the solution space of the differential equation, and only a partial moving frame can be constructed. In this case, this is a reflection of the automorphic property of (52) with respect to the pseudogroup action (120). A more detailed investigation of the group foliation method in the context of partial moving frames could be of interest.

(c) The systematic construction of Bäcklund transformations using symmetry reduction of exterior differential systems introduced by Anderson and Fels applies only to Lie groups. The group foliation/inductive moving frames approach outlined in Section 6 does not have this limitation. It would be worthwhile to pursue the possibility of constructing new Bäcklund transformations by realizing systems of interest as resolving systems for infinite-dimensional Lie pseudogroups; these ideas are also most likely closely related to the reduction methods for infinite-dimensional Lie pseudogroups introduced by Pohjanpelto [48]. The investigation of nonmaximal rank resolving systems could also produce interesting examples. Finally, the group foliation algorithm in conjunction with inductive moving frames may provide a means for constructing coverings of differential equations $[16,20]$.

(d) Through the use of joint moving frames and joint invariants [37, 38], the moving frames approach to group foliation may be adapted to finite difference equations. This adaptation is the subject of a work in progress [53]. Investigating the possibility of discrete group foliation as a numerical method for solving differential equations could be fruitful.

It may also be worthwhile to pursue the construction of Bäcklund transformations for finite difference or differential difference equations and compare these results with similar notions from discrete differential geometry and integrable systems $[4,10]$.

(e) In the case of group foliation by finite-dimensional Lie group actions, nonmaximal rank resolving systems correspond to what are called partially invariant solutions [47]. The question of when a partially invariant solution 
is irreducible, that is, not obtainable as an invariant or partially invariant solution for a subgroup, has been studied by Ondich [45]. This allows for an extension of the classification of group invariant solutions [35] to partially invariant solutions. It may be interesting to investigate the extension of such a classification and the notion of irreducibility in the context of group foliation.

(f) Invariant submanifold flows find applications in a diversity of fields such as control theory [33], elasticity theory [24], and computer vision [8, 50], and it is possible that the idea of invariant flow reconstruction presented in [52] may provide insight in some of these areas. Theoretical application of invariant flow reconstruction is also worth exploring. For example, Mansfield and van der Kamp [29] have studied the question of when the integrability (in the sense of possessing infinitely many symmetries) of a differential invariant signature flow 'lifts' to integrability of the flow itself. We suspect that their results could be reinterpreted within our framework.

\section{Acknowledgements}

The authors would like to thank Mark Fels, Juha Pohjanpelto, and Peter Olver for stimulating discussions of different aspects of this work. We also would like to thank the referees for corrections and comments that helped improve the exposition. The first author's research was done in part at the University of Minnesota, supported by NSF Grant DMS 1108894, and at Harvey Mudd College, supported by NSF Grant DMS 0839966.

\section{References}

[1] I. M. Anderson, 'The variational bicomplex', Technical Report, Utah State University, 2000.

[2] I. M. Anderson and M. E. Fels, 'Transformations of Darboux integrable systems', in Differential Equations: Geometry, Symmetries and Integrability, Abel Symp., 5 (Springer, Berlin, 2009), 21-48.

[3] I. A. Anderson and M. E. Fels, 'Exterior differential systems with symmetry', Acta Appl. Math. 87 (2005), 3-31.

[4] A. I. Bobenko and Y. B. Suris, Discrete Differential Geometry: Integrable Structure, Graduate studies in Mathematics, 98 (American Mathematical Society, Providence, RI, 2008).

[5] F. Calogero, 'A solvable nonlinear wave equation', Stud. Appl. Math. 70(3) (1984), 189-200.

[6] É. Cartan, 'Les groupes de transformations continus, infinis, simples', Ann. Éc. Norm. 26 (1909), 93-161.

[7] B. Champagne and P. Winternitz, 'On the infinite-dimensional symmetry group of the Davey-Stewartson equations', J. Math. Phys. 29(1) (1988), 1-8.

[8] T. F. Chan and J. Shen, 'Non-texture inpainting by curvature-driven diffusions', J. Visual Comm. Image Rep. 12 (2001), 436-449. 
[9] Y. A. Chirkunov and V. Y. Prudnikov, 'Group reduction of the Lamé equations', Prikl. Mat. Mekh. 52(3) (1988), 366-371.

[10] A. A. Coley, (ed.) Bäcklund and Darboux Transformations: The Geometry of Solitons: AARMS-CRM Workshop, June 4-9, 1999, Halifax, NS, Canada, Vol. 29, American Mathematical Society, 2001.

[11] D. David, D. Levi and P. Winternitz, 'Bäcklund transformations and the infinite-dimensional symmetry group of the Kadomtsev-Petviashvili equation', Phys. Lett. A 118(8) (1986), 390-394.

[12] M. Faucher and P. Winternitz, 'Symmetry analysis of the Infeld-Rowlands equation', Phys. Rev. E 48 (1993), 3066-3071.

[13] M. Fels and P. J. Olver, 'Moving coframes. II. Regularization and theoretical foundations', Acta Appl. Math. 55 (1999), 127-208.

[14] H. G. Guggenheimer, Differential Geometry, (McGraw-Hill, New York, 1963).

[15] E. Hubert, 'Differential invariants of a Lie group action: syzygies on a generating set', J. Symbolic Comput. 44(4) (2009), 382-416.

[16] S. Igonin, 'Coverings and fundamental algebras for partial differential equations', J. Geom. Phys. 56(6) (2006), 939-998.

[17] V. Itskov, P. J. Olver and F. Valiquette, 'Lie completion of pseudo-groups', Transform. Groups 16 (2011), 161-173.

[18] N. Jacobson, Lie Algebras, (Dover, New York, 1979).

[19] H. H. Johnson, 'Classical differential invariants and applications to Partial differential equations', Math. Ann. 148 (1962), 308-329.

[20] I. S. Krasil'shchik and A. M. Vinogradov, 'Nonlocal symmetries and the theory of covering', Acta Appl. Math. 2 (1984), 79-86.

[21] B. Kruglikov and V. Lychagin, 'Invariants of pseudo-group actions: homological methods and finiteness theorem', Int. J. Geom. Methods Mod. Phys. 3 (2006), 1131-1165.

[22] B. Kruglikov and V. Lychagin, 'Global Lie-Tresse theorem', 2015, arXiv:1111.5480v3.

[23] A. Kumpera, 'Invariants différentiels d'un pseudo-groupe de Lie', J. Differ. Geom. 10 (1975), 289-416.

[24] J. Langer and D. A. Singer, 'Lagrangian aspects of the Kirchhoff elastic rod', SIAM Rev. 38(4) (1996), 605-618.

[25] H.-C. Lei, 'Group splitting and linearization mapping of a solvable non-linear wave equation', Int. J. Nonlinear Mech. 33(3) (1998), 461-471.

[26] S. Lie and G. Scheffers, Vorlesungen über Continuierliche Gruppen mit Geometrischen und Anderen Anwendungen, (B. G. Teubner, Leipzig, 1893).

[27] S. Lie, 'Zur allgemeinen Theorie der partiellen Differentialgleichungen beliebiger Ordnung', Leipz. Ber. 1 (1895), 53-128.

[28] E. L. Mansfield, A Practical Guide to the Invariant Calculus, Cambridge Monographs on Applied and Computational Mathematics, (Cambridge University Press, Cambridge, 2010).

[29] E. L. Mansfield and P. H. van der Kamp, 'Evolution of curvature invariants and lifting integrability', J. Geom. Phys. 56(8) (2006), 1294-1325.

[30] L. Martina, M. B. Sheftel and P. Winternitz, 'Group foliation and non-invariant solutions of the heavenly equation', J. Phys. A 34 (2001), 9243-9263.

[31] P. Medolaghi, 'Classificazione delle equazioni alle derivative parziali del secondo ordine, che ammettono un gruppo infinito di trasformazioni puntuali', Ann. Mat. Pura Appl. 31898001 (1898), 229-263.

[32] J. Munoz, F. J. Muriel and J. Rodriguez, 'On the finiteness of differential invariants', J. Math. Anal. Appl. 284 (2003), 266-282. 
[33] M. Niethammer, A. Tannenbaum and S. Angenent, 'Dynamic active contours for visual tracking', IEEE Trans. Automat. Control 51 (2006), 562-579.

[34] Y. Nutku and M. B. Sheftel, 'Differential invariants and group foliation for the complex Monge-Ampère equation', J. Phys. A 34 (2001), 137-156.

[35] P. J. Olver, Applications of Lie Groups to Differential Equations, 2nd edn, (Springer, New York, 1993).

[36] P. J. Olver, Equivalence, Invariants, and Symmetry, (Cambridge University Press, Cambridge, 1995).

[37] P. J. Olver, 'Moving frames and joint differential invariants', Regul. Chaotic Dyn. 4(4) (1999), $3-18$.

[38] P. J. Olver, 'Joint invariant signatures', Found. Comput. Math. 1 (2001), 3-67.

[39] P. J. Olver, 'Generating differential invariants', J. Math. Anal. Appl. 333 (2007), 450-471.

[40] P. J. Olver, 'Lectures on moving frames', in Symmetries and Integrability of Difference Equations, (eds. D. Levi, P. Olver, Z. Thomova and P. Winternitz) London Mathematical Society Lecture Note Series, 381 (Cambridge University Press, Cambridge, 2011), 207-246.

[41] P. J. Olver and J. Pohjanpelto, 'Maurer-Cartan forms and the structure of Lie pseudo-groups', Selecta Math. 11 (2005), 99-126.

[42] P. J. Olver and J. Pohjanpelto, 'Differential invariants for Lie pseudo-groups', Comput. Appl. Math. 1 (2007), 1-27.

[43] P. J. Olver and J. Pohjanpelto, 'Moving frames for Lie pseudo-groups', Can. J. Math. 60 (2008), 1336-1386.

[44] P. J. Olver and J. Pohjanpelto, 'Differential invariant algebras of Lie pseudo-groups', Adv. Math. 222 (2009), 1746-1792.

[45] J. Ondich, 'The reducibility of partially invariant solutions of systems of partial differential equations', Eur. J. Math. 6(4) (1995), 329-354.

[46] P. J. Olver and F. Valiquette, 'Recursive moving frames for Lie pseudo-groups', Preprint SUNY New Paltz, 2014.

[47] L. V. Ovsiannikov, Group Analysis of Differential Equations, (Academic Press, New York, 1982).

[48] J. Pohjanpelto, 'Reduction of exterior differential systems with infinite dimensional symmetry groups', BIT Num. Math. 48 (2008), 337-355.

[49] A. A. M. Rodrigues, 'On infinite Lie groups', Ann. Inst. Fourier 31(3) (1981), 245-274.

[50] G. Sapiro, Geometric Partial Differential Equations and Image Analysis, (Cambridge University Press, Cambridge, 2001).

[51] O. Stormark, Lie's Structural Approach to PDE Systems, Encyclopedia of Mathematics and its Applications, 80 (Cambridge University Press, New York, 2000).

[52] R. Thompson, 'Applications of moving frames to group foliation of differential equations', PhD Thesis, University of Minnesota, 2013.

[53] R. Thompson and F. Valiquette, 'Group foliation of finite difference equations' (in preparation).

[54] A. Tresse, 'Sur les invariants différentiels des groupes continus de transformations', Acta Math. 18 (1894), 1-88.

[55] F. Valiquette, 'Solving local equivalence problems with the equivariant moving frame method', SIGMA 9 (2013), 029.

[56] E. Vessiot, 'Sur l'intégration des systèmes différentiels qui admettent des groupes continus de transformations', Acta Math. 28 (1904), 307-349.

[57] S.-T. Yau, 'Calabi's conjecture and some new results in algebraic geometry', Proc. Natl Acad. Sci. USA 74(5) (1977), 1798-1799. 